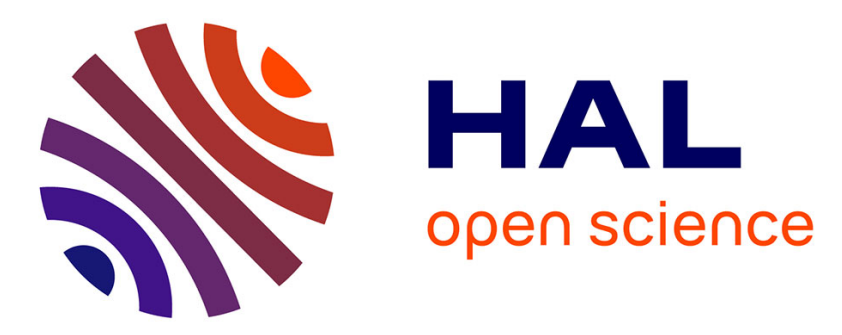

\title{
Wall Model and Mesh Influence Study for Partial Cavities
}

\author{
Eric Goncalvès da Silva, Jean Decaix
}

\section{To cite this version:}

Eric Goncalvès da Silva, Jean Decaix. Wall Model and Mesh Influence Study for Partial Cavities. European Journal of Mechanics - B/Fluids, 2012, 31, pp.12-29. 10.1016/j.euromechflu.2011.08.002 . hal-00620742

\section{HAL Id: hal-00620742 \\ https://hal.science/hal-00620742}

Submitted on 20 Dec 2012

HAL is a multi-disciplinary open access archive for the deposit and dissemination of scientific research documents, whether they are published or not. The documents may come from teaching and research institutions in France or abroad, or from public or private research centers.
L'archive ouverte pluridisciplinaire HAL, est destinée au dépôt et à la diffusion de documents scientifiques de niveau recherche, publiés ou non, émanant des établissements d'enseignement et de recherche français ou étrangers, des laboratoires publics ou privés. 


\title{
Wall Model and Mesh Influence Study for Partial Cavities
}

\author{
Eric Goncalvès*, Jean Decaix* \\ LEGI-Grenoble INP, 1025 rue de la Piscine, 38400 St Martin d'Heres, France
}

\begin{abstract}
This numerical work investigates the mesh influence in the near-wall area when a partial cavity develops and interacts with the turbulent boundary layer. Different meshes varying by the first cell size are compared, and unsteady simulations are performed with a two-phase one-fluid RANS solver. Numerical results are given for two Venturi geometries (quasi stable and unstable behaviour) and comparisons are made with experimental data.

Keywords: cavitation, homogeneous model, RANS simulations, wall functions, turbulence model
\end{abstract}

\section{Introduction}

Cavitation is a significant engineering phenomenon that occurs in fluid machinery, fuel injectors, marine propellers, nozzles, underwater bodies, etc. In most cases, cavitation is an undesirable phenomenon, significantly degrading performance, resulting in reduced flow rates, lower pressure increases in pumps, load asymmetry, vibrations, noise and erosion.

\footnotetext{
*Corresponding author.

Email address: Eric.Goncalves@legi.grenoble-inp.fr (Eric Goncalvès )
} 
A considerable efforts has been realized to understand the fundamental physics of cavitation phenomena concerning the development of partial sheet cavities. Sheet cavitation that appear on solid bodies are characterized by a closure region which always fluctuates, with the presence of a re-entrant jet. This jet is mainly composed of liquid which flows upstream along the solid surface. Partial cavitation can be classified as either closed or open attached cavitation, depending on the flow in the cavity closure region. A closed partial cavity has a relatively stable cavity length and a cavity closure that is relatively free of bubbles. An open cavity is typically frothy in appearance, and has a periodically varying length that is associated with the shedding of vapour clouds [1]. Both types of cavities have been studied, experimentally and numerically, to describe and investigate the transition between stable and unstable behaviour, the development of the re-entrant jet, the interaction with the turbulent boundary layer, and the mechanism of cloud cavitation generation $[2,3,4]$.

Cavitating flows are challenging to model and simulate, since they are turbulent, highly dynamic and highly unstable two-phase flows. Several numerical models have been developed to investigate such cavitating flows, especially with one-fluid RANS solvers. The homogeneous mixture model treats the cavitating flows as a mixture of two fluids behaving as one. These models are based on the assumption of local kinematic equilibrium between phases (the local velocity is the same for both phases), local thermal and mechanic equilibrium between the two components (local temperature and pressure 
equality between phases). Moreover, vaporization or condensation processes are assumed to be instantaneous. This model cannot reproduce strong thermodynamic or kinetic non equilibrium effects but, because of its simplicity, it is often used for numerical simulations $[5,6,7,8,9,10,11,12]$.

In most applications, wall functions are used as wall boundary conditions to reduce the simulation cost. It is usually assumed that standard single-phase wall functions (a two-layer model based on a linear and logarithmic velocity profile) are available in a mixture liquid/vapour flow. The validity of this assumption was not attested. Experimental works investigated the modifications of turbulent properties and the evolution of the logarithmic law in the wake of a partial stable cavity developing on a NACA0015 hydrofoil [13]. They showed that the presence of the cavity influences the boundary layer structure even far from the closure of the cavity. For computations integrated down to the wall with fine meshes, it is usually assumed that the damping functions of turbulence models established in the case of single-phase flows are available in a two-phase cavitating flow. The wall model influence regarding the re-entrant jet development and modifications introduced in the boundary layer is very complex to study. This fact is linked with the experimental difficulties that arise when velocity measurements must be performed in bubbly mixtures near the wall.

The present work is devoted to a numerical study of two partial cavities appearing on Venturi geometries. A particular emphasis is placed on the study of the wall treatment and the mesh refinement near the wall. An in-house finite-volume code solving the Reynolds-Averaged Navier-Stokes (RANS) 
compressible equations was developed with a homogeneous approach [14, 15, 16]. The cavitation phenomenon is modelled by a barotropic liquid-vapour mixture equation of state (EOS). Different meshes are built and tested in which the $y^{+}$values in the first adjacent cells vary. Various unsteady simulations are performed with and without a two-layer wall law. Moreover, a new wall treatment is proposed and compared with standard wall functions. This wall model is based on the local integration of the thin boundary layer equations (TBLE). With this wall treatment the pressure gradient is accounted, and no assumptions are done on the velocity profile, especially the validity in both unsteady and mixture liquid/vapour flows. Our goals in this study are:

- To compare fine-mesh and wall-functions simulations regarding the sheet cavity dynamics, and to evaluate the influence of the wall treatment with and without wall functions.

- To investigate the behaviour of the standard two-layer wall functions through a mesh refinement study.

- To compare two different wall functions: TBLE and two-layer models. This would help in evaluating the standard law of the wall in unsteady cavitating flows.

In this paper, we will first review the theoretical formulation, including physical models, wall functions and elements of the numerical methods. This is followed by sets of results on Venturi geometries and discussions. 


\section{Numerical tool}

The numerical simulations are carried out using an in-house CFD code solving the one-fluid compressible RANS system.

The homogeneous mixture model assumes that the two phases are strongly coupled and moving at the same velocity. The phases are assumed to share the same temperature $T$ and the same pressure $P$. The evolution of the twophase flow can be described by the conservation equations that employ the representative flow properties as unknowns just as in a single-phase problem. The mixture density $\rho$ is defined by:

$$
\rho=\alpha \rho_{V}+(1-\alpha) \rho_{L}
$$

where $\rho_{L}$ and $\rho_{V}$ are respectively the liquid and vapour densities. The void ratio $\alpha$ characterizes the volume of vapour in each cell: $\alpha=1$ means that the cell is completely filled by vapour and inversely, a complete liquid cell is represented by $\alpha=0$. Liquid and vapour phases are characterized by their thermodynamic properties. On each cell, the unknowns are calculated by averaging them over the volume occupied.

\subsection{Reynolds-Averaged Navier-Stokes equations}

For turbulent computations, the Reynolds-averaged compressible equations are used, coupled with two-equation turbulence models. For low Mach number applications, an inviscid preconditioning method is necessary [17, 18], based on the modification of the derivative term by a pre-multiplication with a suitable preconditioning matrix $P_{c}$. These equations can be expressed as:

$$
P_{c}^{-1} \frac{\partial w}{\partial t}+\operatorname{div}\left(F_{c}-F_{v}\right)=S
$$




$$
w=\left(\begin{array}{c}
\rho \\
\rho V \\
\rho E \\
\rho k \\
\rho \Psi
\end{array}\right) \quad ; F_{c}=\left(\begin{array}{c}
\rho V \\
\rho V \otimes V+p \overline{\bar{I}} \\
(\rho E+p) V \\
\rho k V \\
\rho \Psi V
\end{array}\right) \quad ; F_{v}=\left(\begin{array}{c}
0 \\
\overline{\overline{\tau^{v}}}+\overline{\overline{\tau^{t}}} \\
\left(\overline{\overline{\tau^{v}}}+\overline{\overline{\tau^{t}}}\right) \cdot V-Q^{v}-Q^{t} \\
\left(\mu+\mu_{t} / \sigma_{k}\right) \operatorname{grad} k \\
\left(\mu+\mu_{t} / \sigma_{\Psi}\right) \operatorname{grad} \Psi
\end{array}\right)
$$

where $w$ denotes the conservative variables, $F_{c}$ and $F_{v}$ the convective and viscous flux densities and $S$ the source terms, which concern only the transport equations. $k$ is the turbulent kinetic energy and $\Psi$ is a turbulent variable. The exact expression of the eddy-viscosity $\mu_{t}$ and the source terms depends on the turbulence model as well as constants $\sigma_{k}$ and $\sigma_{\Psi}$.

The total stress tensor $\overline{\bar{\tau}}$ is evaluated using the Stokes hypothesis, Newton's law and the Boussinesq assumption. The total heat flux vector $Q$ is obtained from the Fourier law involving a turbulent thermal conductivity $\lambda_{t}$ with the constant Prandtl number hypothesis.

$$
\begin{aligned}
\overline{\bar{\tau}} & =\overline{\overline{\tau^{v}}}+\overline{\overline{\tau^{t}}}=\left(\mu+\mu_{t}\right)\left[\left(\operatorname{grad} V+(\operatorname{grad} V)^{t}\right)-\frac{2}{3}(\operatorname{div} V) \overline{\bar{I}}\right]+\frac{2}{3} \rho k \overline{\bar{I}} \\
Q & =Q^{v}+Q^{t}=-\left(\lambda+\lambda_{t}\right) \operatorname{grad} T \quad \text { with } \quad \lambda_{t}=\frac{\mu_{t} C_{p}}{P_{r t}}
\end{aligned}
$$

In pure liquid, the viscosity $\mu$ is determined by an exponential law and, in pure vapour, the viscosity follows the Sutherland law. The mixture viscosity is defined as the arithmetic mean of the liquid and vapour viscosities (fluctuations of viscosity are neglected) [19]:

$$
\mu_{L}(T)=\mu_{0_{L}} \exp (B / T)
$$




$$
\begin{aligned}
\mu_{V}(T) & =\mu_{0_{V}} \sqrt{\frac{T}{293}} \frac{1+T_{S} / 293}{1+T_{S} / T} \\
\mu(T, \alpha) & =\alpha \mu_{V}(T)+(1-\alpha) \mu_{L}(T)
\end{aligned}
$$

where $\mu_{0_{L}}, \mu_{0_{V}}, B$ and $T_{S}$ are constants.

The mixture thermal conductivity $\lambda$ is also defined as the arithmetic mean of the liquid and vapour values:

$$
\lambda(\alpha)=\alpha \frac{\mu_{V} C_{p_{V}}}{P_{r_{V}}}+(1-\alpha) \frac{\mu_{L} C_{p_{L}}}{P_{r_{L}}}
$$

The turbulent Prandtl number $P_{r t}$ is set to 1 .

\subsection{The cavitation model}

To close the system, an equation of state (EOS) is necessary to link the pressure to the thermodynamic variables. Pure phases follow the stiffened gas EOS. The barotropic law proposed by Delannoy [20] is considered for the mixture.

This law is characterized by its maximum slope $1 / c_{\text {baro }}^{2}$. The quantity $c_{\text {baro }}$ is an adjustable parameter of the model, which can be interpreted as the minimum speed of sound in the mixture.

When the pressure is between $P_{v a p}+\Delta P$ and $P_{v a p}-\Delta P$, the following relationship applies:

$$
P(\alpha)=P_{v a p}+\left(\frac{\rho_{L}^{s a t}-\rho_{V}^{s a t}}{2}\right) c_{b a r o}^{2} \operatorname{Arcsin}(1-2 \alpha)
$$

where $\Delta P$ represents the pressure range of the law and, for a void ratio value of 0.5 , the pressure is equal to the saturation pressure $P_{v a p}$. This law introduces a small non-equilibrium effect on the pressure. The cavitation 
phenomenon is assumed to be isothermal, therefore there is no coupling with the temperature.

The hyperbolicity and convexity of the EOS have been demonstrated in [14]. The influence of $c_{\text {baro }}$ has been studied in previous works. In the present paper, the value of $c_{\text {baro }}$ is set to $0.47 \mathrm{~m} / \mathrm{s}$, corresponding to a pressure range of $\Delta P=175 \mathrm{~Pa}$.

\subsection{The turbulence modelling}

In the present study, various transport-equation turbulence models were used: the one-equation model proposed by Spalart and Allmaras (SA) [21, 22], the Smith $k-\ell$ turbulence model (KL) [23, 24], the Menter SST model (KWSST) $[25,26]$ and the Jones-Launder $k-\varepsilon$ model (KE) [27].

Turbulence models always lead to the generation of stable cavities, because very strong turbulent eddy-viscosity $\mu_{t}$ inside the cavity prevents the formation of the re-entrant jet which plays a major role in driving the instability of partial sheet cavity. The link to compressibility effects on turbulence is not clear. DNS of the supersonic boundary layer demonstrated a reduction in $k$ production as a consequence of compressibility $[28,29,30]$. In cavitating flows, the supersonic regime is reached in the mixture area because of the drastic diminution of the speed of sound. The detailed mechanisms of the interaction between turbulent flows and cavitation have not yet been clearly revealed, especially for phenomena occurring at small scales.

To limit the turbulent viscosity, one can use an eddy-viscosity limiter in the mixture area. In the present study, we used the Reboud correction [31] spe- 
cially developped for the two-phase flow. This limiter introduces a function $f(\rho)$ in the computation of the turbulent viscosity for the $k-\varepsilon$ model:

$$
\mu_{t}=f(\rho) C_{\mu} \frac{k^{2}}{\varepsilon} \quad \text { with } f(\rho)=\rho_{V}+(1-\alpha)^{n}\left(\rho_{L}-\rho_{V}\right)
$$

where $n$ is a parameter set to 10 .

This correction is extended to other turbulence models with the same function $f(\rho)$.

\subsection{Wall functions}

\subsubsection{Two-layer wall functions}

At the wall, a two-layer approach is used:

$$
\begin{aligned}
u^{+}=y^{+} & \text {if } & y^{+}<11.13 \\
u^{+}=\frac{1}{\kappa} \ln y^{+}+5.25 & \text { if } & y^{+}>11.13 \\
u^{+}=\frac{u}{U_{\tau}} ; \quad y^{+}=\frac{y U_{\tau}}{\nu_{w}} \quad ; & U_{\tau}=\sqrt{\frac{\tau_{w}}{\rho_{w}}} &
\end{aligned}
$$

where $\kappa=0.41$ is the von Karman constant and the subscript $w$ indicates wall values. We assume that wall functions are similar in a two-phase flow and in a single-phase flow. For unsteady flows, the existence of a wall law is assumed to be valid at each instant.

With regard to the turbulent transport-equation models, the production of $k$ is computed according to the formulation proposed by Viegas and Rubesin [32]. The value of $\ell$ in the first cell is computed with the linear relation $l=\kappa y$.

For the one-equation Spalart-Allmaras model, the transported quantity is 
calculated using the model's closure relations, the velocity profile and a mixing-length formulation for the eddy-viscosity. More details concerning the wall law approach are given in [33].

\subsubsection{Thin boundary layer equations}

The logarithmic law implies an implicit integration of the averaged boundarylayer equations from the first grid point to the wall, under the assumption of constant stress, with a mixing-length model to parameterize turbulent transport [34]. Since two decades, the validity of the logarithmic law in turbulent boundary layer with or without pressure gradient is challenged $[35,36]$. Mainly, the parameters of the logarithmic law seem to be dependent on the geometry flow and the Reynolds number. Moreover, the low bound of the logarithmic law is not universal. In the presence of strong adverse pressure gradients leading to separation, the assumption of the existence of a logarithmic law does not hold. This prompted the development of models for large-eddy simulations (LES) based on the integration of a simplified averaged set of partial equations derived from the NavierStokes equations. This set of equations, known as the thin-boundary-layer equations (TBLE), is:

$$
\frac{\partial u_{i}}{\partial t}+\frac{\partial u_{i} u_{j}}{\partial x_{j}}+\frac{1}{\rho} \frac{d P}{d x_{i}}=\frac{\partial}{\partial y}\left[\left(\mu+\mu_{t}\right) \frac{\partial u_{i}}{\partial y}\right]
$$

In this equation, $u_{i}$ is the mean velocity in direction $x_{i}$ and $y$ indicates the direction normal to the wall. A fine one-dimensional grid is embedded between the first grid point and the wall, and the turbulent boundary-layer equations are solved in the embedded mesh. The outer-layer LES provides the boundary condition for the inner layer, whereas the inner-layer calculation provides the wall stress required by the LES. Various features of this 
model were explored [37, 38, 39]. Starting from Eq. (11), Wang and Moin [39] compared two simpler models to a full TBLE model: first, totally neglecting the left-hand side and second including only the streamwise pressure gradient. They showed that the inclusion of the pressure gradient allows for a significant improvement of the model predictions.

In the present work, we reduce the left-hand side of the equation, written for the mixture, by neglecting the convection term. The simplified mean streamwise momentum equation can be integrated analytically in the wall normal direction:

$$
\left(\mu+\mu_{t}\right) \frac{\partial u}{\partial y}-y \frac{\partial P}{\partial x}=\tau_{w}
$$

To solve this equation, a virtual fine mesh is embedded between the wall and the first mesh point. The first cell-center is subdivided with $N$ nodes, regularly located with a step $\Delta y$. All derivatives are computed with a secondorder finite difference scheme. The viscosity $\mu$ and the density $\rho$ are assumed to be constant in the embedded grid. The discretized equation written on the point $i+1 / 2$ is:

$$
\left(\mu_{e f f_{i}}+\mu_{e f f_{i+1}}\right)\left(\frac{u_{i+1}-u_{i}}{\Delta y}\right)=\frac{\mu_{e f f_{1}}+\mu_{e f f_{2}}}{\Delta y} u_{2}+2 y_{i} \frac{d P}{d x} \quad \text { with } \quad \mu_{e f f}=\mu+\mu_{t}
$$

The turbulent viscosity $\mu_{t}$ is given as a function of $k$ and $l$ through the relation of the Smith model:

$$
\mu_{t}=\mu \chi f_{\mu} \quad ; \quad \chi=\frac{\rho \sqrt{2 k} l}{\mu B_{1}^{1 / 3}} \quad ; \quad B_{1}=18
$$




$$
f_{\mu}=\left(\frac{25.5^{4} f_{1}+2^{2} \chi^{2}+\chi^{4}}{25.5^{4}+2^{2} \chi^{2}+\chi^{4}}\right)^{1 / 4} \quad ; \quad f_{1}=\exp \left(-50\left(\frac{l}{\kappa y}\right)^{2}\right)
$$

An analytical expression of $k$ can be expressed with the assumptions of boundary-layer flow, convection neglected and a linear evolution for the length scale near the wall $l=\kappa y[24]$ :

$$
\begin{aligned}
4 \rho^{2} k^{2} & =a y^{\sqrt{p}}-\frac{A}{p}+\frac{B}{1-p} y+\frac{C}{4-p} y^{2}+\frac{4 \rho_{N}^{2} k_{N}^{2}}{4-p}\left(\frac{y}{y_{N}}\right)^{2} \\
p & =\frac{4 \sigma_{k}}{\kappa^{2} B_{1}} \quad ; \quad A=-B_{1}^{4 / 3} p \tau_{w}^{2} \quad ; \quad B=-2 B_{1}^{4 / 3} p \tau_{w} \frac{d P}{d x} \\
C & =-B_{1}^{4 / 3} p\left(\frac{d P}{d x}\right)^{2}+\left(\frac{\rho q^{2}\left(y_{N}\right)}{y_{N}}\right)^{2}
\end{aligned}
$$

The integration is performed using a Newton algorithm for the variable $\tau_{w}$. For the first iteration, the value $\tau_{w}{ }^{(0)}$ is evaluated with the two-layer wall functions. In our calculations, the number of nodes $N$ necessary to solve TBLE were fixed at 30, which seems to be sufficient for high Reynolds number simulations. The influence of this number was not studied.

For the turbulent quantities, the formulation for the length scale $l$ is similar to the two-layer wall functions treatment. The production of the turbulent kinetic energy $P_{k}$ is evaluated following the Viegas and Rubesin formulation:

$$
P_{k}=\frac{1}{y_{N}} \int_{0}^{y_{N}} \tau_{x y}^{t} \frac{\partial u}{\partial y} d y
$$

where $\tau_{x y}^{t}$ is the turbulent stress tensor component, which can be expressed with the wall shear stress and the pressure gradient:

$$
P_{k}=\frac{1}{y_{N}} \int_{0}^{y_{N}} \frac{\mu_{t}}{\left(\mu+\mu_{t}\right)^{2}}\left(\tau_{w}+y \frac{d P}{d x}\right)^{2} d y
$$

This integral is computed with the trapezoidal rule. 


\subsection{Numerical methods}

The numerical simulations are carried out using an implicit CFD code solving the RANS/turbulent systems for multi-domain structured meshes. This solver is based on a cell-centered finite-volume discretization.

\subsubsection{Spatial discretization}

For the mean flow, the convective flux density vector on a cell face is computed with the Jameson scheme [40]. The artificial viscosity includes a second-order dissipation term $D_{2}$ and a fourth-order dissipation term $D_{4}$, which involve two tunable parameters $k^{(2)}$ and $k^{(4)}$. To take into consideration the mesh deformation, a weighted scheme is implemented. Indeed, as shown in Figure 4, the second adjacent cell to a wall can be largely finer than the first one. This important change of cell size induces a loss of spatial accuracy, which can be corrected. The centered numerical fluxes and the gradient computations are corrected by using a weighted discretization operator $\tilde{\mu} w_{i+1 / 2}$ instead of the classical operator $\mu w_{i+1 / 2}=0.5\left(w_{i+1}+w_{i}\right)$. Let $A$ and $B$ two points and $M$ an interior point of the segment $A B$, the weighted discrete operator is defined by:

$$
\tilde{\mu}_{A}^{B} w_{M}=\frac{M B}{A B} w_{B}+\frac{A M}{A B} w_{A}
$$

The viscous terms are discretized by a second-order space-centered scheme. For the turbulence transport equations, the upwind Roe scheme [41] is used to obtain a more robust method. The second-order accuracy is obtained by introducing a flux-limited dissipation [42]. 


\subsubsection{Temporal discretization}

Time integration is achieved using a low-cost implicit method [43]. The implicit method consists in solving, at each time step, a system of equations arising from the linearization of a fully implicit scheme. The main advantage of this method is that the storage of the Jacobian matrix is completely eliminated, which leads to a low-storage algorithm. More details are given in $[14]$.

For the turbulence transport equations, the diffusive flux Jacobian matrix is replaced by its spectral radius. The source term needs special treatment [44]. Only the negative part of the source term Jacobian matrix is considered and replaced by its spectral radius. The system obtained is solved with a linealternated Jacobi relaxation algorithm.

\subsubsection{Inlet and outlet boundary conditions}

The numerical treatment of boundary conditions is based on the use of the preconditioned characteristic relationships [14]. We assume that inlet and outlet areas are in a pure liquid region; no cavitation appears in these boundaries.

\section{Computational results for a quasi-stable partial cavity}

\subsection{Experimental conditions [45]}

The Venturi was tested in the cavitation tunnel of the CREMHyG (Centre d'Essais de Machines Hydrauliques de Grenoble). It is characterized by a divergence angle of $4^{\circ}$, illustrated in Fig. 1 . The edge forming the throat of the Venturi is used to fix the separation point of the cavitation cavity. This 
geometry is equipped with five probing holes to allow various measurements such as the local void ratio, instantaneous local speed and wall pressure (Fig. 1).

The selected operating point is characterized by the following physical parameters [45]:

$U_{\text {inlet }}=10.8 \mathrm{~m} / \mathrm{s}$, the inlet velocity

$\sigma_{\text {inlet }}=\frac{P_{\text {inlet }}-P_{\text {vap }}}{0.5 \rho U_{\text {inlet }}^{2}} \simeq 0.55$, the cavitation parameter in the inlet section $T_{\text {ref }} \simeq 293 \mathrm{~K}$, the reference temperature

$L_{r e f}=252 \mathrm{~mm}$, the reference length, which corresponds to the chord of a blade of a turbomachinery.

$R e_{L_{r e f}}=\frac{U_{\text {inlet }} L_{r e f}}{\nu}=2.710^{6}$, the Reynolds number

With these parameters, a cavity length $L$ ranging from $70 \mathrm{~mm}$ to $85 \mathrm{~mm}$ is obtained. The experimental views for this geometry show a relatively stable cavity behaviour (see Fig. 2). It is characterized by an almost constant length, although the closure region always fluctuates, with the presence of a re-entrant jet and little vapour cloud shedding. For this geometry, no periodic cycles with large shedding were observed.

\subsection{Meshes}

The initial grid is a H-type topology. It contains 251 nodes in the flow direction and 62 nodes in the orthogonal direction. A special contraction of the mesh is applied in the main flow direction just after the throat to better simulate the two-phase flow area (Fig. 3). The $y^{+}$values of the mesh, at the center of the first cell, vary between 12 and 27 for a non cavitating computation. 
From this grid, four meshes are built by adding or eliminating cells near the wall. A view of all meshes near the throat is illustrated in Figure 4. The $y^{+}$ values are presented in Figure 5 obtained from a non cavitating computation with the Spalart-Allmaras turbulence model. With the finest mesh, $251 \times 81$, the $y^{+}$values are close to 1 , and all computations are performed without wall functions. For all other coarse meshes, wall functions are used as wall boundary conditions.

\subsection{Numerical parameters}

For the non cavitating regime, computations are started from a uniform flowfield using a local time step. The numerical parameters used are:

- the CFL number, 10

- Jacobi iterations for the implicit stage, 15

- the two coefficients of the artificial dissipation, $k^{(2)}=0$ and $k^{(4)}=0.032$

- the farfield value of turbulent kinetic energy, $k_{\infty}=0.0045 \mathrm{~m}^{2} / \mathrm{s}^{2}$

- the farfield value of length, $l_{\infty}=1.410^{-6} \mathrm{~m}$

- the farfield value of specific dissipation $\omega_{\infty}=45000 \mathrm{~s}^{-1}$

For the unsteady cavitating regime, computations are performed with the dual time stepping method and are started from the non cavitating numerical solution. The numerical parameters are:

- the dimensionless time step, $\Delta t^{*}=\frac{\Delta t U_{\text {inlet }}}{L_{\text {ref }}}=9.7510^{-3}$

- sub-iterations of the dual time stepping method, 100

- the CFL number, 0.5

- Jacobi iterations for the implicit stage, 15

- the two coefficients of the artificial dissipation, $k^{(2)}=1$ and $k^{(4)}=0.045$. 


\subsection{Comparison with and without wall functions}

Two meshes are used for these comparisons: the finest mesh $251 \times 81$ computed without wall functions, and the coarse mesh $251 \times 62$ computed with the two-layer wall functions. All results obtained with this coarse mesh have been presented in [16]. Various turbulence models are considered: the Spalart-Allmaras, Smith and Jones-Launder models associated with the Reboud correction and the Menter SST model.

All global unsteady results are summarized in Table (1). The goal is to obtain a quasi-stable sheet cavity whose time-averaged length $L_{\text {cav }}$ varies between 70 and $85 \mathrm{~mm}$ and with a re-entrant jet. The time of simulation is 2 seconds. For all calculations, the cavitation parameter in the inlet section $\sigma_{\text {inlet }}$ is over-estimated in comparison with the experimental value. Moreover, for all turbulence models, the $\sigma_{\text {inlet }}$ value decreases when using the coarse mesh.

\subsubsection{Velocity and void ratio profiles}

The local analysis involves void ratio and velocity profile comparisons inside the cavity. The experimental void ratio and velocity profiles are obtained for five stations by a double optical probe (Fig. 1). The velocity is evaluated as the most probable value and the void ratio is obtained from the signal of the double optical probe using a post-processing algorithm. The relative uncertainty on the void ratio measurement was estimated at roughly $15 \%$ [45]. All numerical values are obtained by a time-averaged treatment.

Figure 6, 7 and 8 show the longitudinal velocity profiles for the experiments and the computations performed with the Spalart-Allmaras model, the Smith 
$k-l$ model and the Jones-Launder $k-\varepsilon$ model, respectively. The overall agreement seems good between the experimental data and the simulations. For stations 1 and 2, no re-entrant jet phenomena occurs in the experiment. Yet, for all results obtained with the fine mesh, at station 2, a re-entrant jet is simulated, especially with the Smith model. Wall function computations do not predict such a phenomenon. Further downstream, for stations 3, 4 and 5, experimental observation indicates a recirculating behaviour with a re-entrant jet extending roughly through half the sheet thickness. According to experiments, this flow configuration is smoothly time fluctuating. This recirculating behaviour with a re-entrant jet is well simulated by all computations. At station 3, the thickness of the recirculating area is very well estimated by the Smith model. At station 5, with the Jones-Launder model, the thickness of the recirculating area is largely over-predicted by the finemesh computation in comparison with the wall functions computation.

Figure 9, 10 and 11 illustrate experimental and numerical results concerning the void ratio profiles, for the same turbulence models. For all computations, the cavity thickness is very well estimated. Discrepancies between the fine mesh and wall functions solutions are weak.

For these three turbulence models, we observe a more extended re-entrant jet computed with the fine mesh. Cavities simulated with wall functions computations are in better agreement with the experimental data. It could be due to the damping functions of turbulence models, calibrated for single-phase flows.

Results obtained with the Menter SST model present a different behaviour. 
Figure 12 shows the longitudinal velocity profiles for the experiments and the computations performed with the Menter SST model. At station 1, both computations provide a similar result. At station 2, the re-entrant jet captured with the wall function computation is too extended. At stations 3 and 4, we observe that the fine-mesh computation is unable to simulate a re-entrant jet. At station 5, a small re-entrant jet is predicted by the finemesh computation. This behaviour with the fine-mesh computation is also observed on the void ratio profiles, illustrated in Figure 13. At stations 3 and 4, the void ratio values are close to unity near the wall. At station 5 , the void ratio values are largely over-estimated in comparison with both experimental and wall function results.

From these calculations performed with the Menter model, it seems that only the wall-function computation provides a partial cavity in acceptable agreement with the experiments. This model does not involve any damping functions. The model calibration should be explored to explain this singular behaviour.

\subsection{Mesh influence with the two-layer wall functions}

Four meshes are used for these comparisons varying by the mesh refinement near the wall in the $y$ direction. All global unsteady results are summarized in Table (2). Except with the $k-\varepsilon$ model, we observe the decrease of the cavitation parameter in the inlet section $\sigma_{\text {inlet }}$ when the mesh becomes coarser. 


\subsubsection{Velocity profiles}

Comparisons are focused on the velocity profiles (from station 2 to 5) and the development of the re-entrant jet.

Figure 14 shows the velocity profiles for the experiments and the computations performed with the Spalart-Allmaras model. Large discrepancies between meshes appear. With the coarsest mesh, $251 \times 59$, the re-entrant jet is not predicted at stations 3 and 4 . Only a small re-entrant jet is simulated at station 5. This mesh is not adapted to capture such partial cavity when the Spalart-Allmaras model is used. With the finest mesh, $251 \times 77$, at station 3 , only a small recirculating area is simulated. The development of the re-entrant jet is not extended enough. For the other intermediate meshes, results are similar at stations 4 and 5 , but at station 3 , the recirculating area obtained with the mesh $251 \times 62$ is in better agreement in comparison with the experimental data. The sensitivity to the near-wall mesh refinement seems important with this turbulence model. Maybe it is due to the effect of the pressure gradient, not explicitly accounted in the wall functions.

Figure 15 plots the velocity profiles for the experiments and the computations performed with the Smith model. At stations 3 to 5, results provided by all meshes are similar and in very close agreement with the experimental values. Discrepancies appear only at station 2 . The mesh $251 \times 77$ is near to predict a recirculating flow. The best result is obtained with the mesh $251 \times 62$.

It seems that cavities simulated with the Smith model are relatively not dependant on the mesh. 
Figure 16 shows the velocity profiles for the experiments and the computations performed with the Jones-Launder model. With the coarsest mesh $251 \times 59$, the re-entrant jet is not predicted at stations 3, 4 and 5. As previously observed with the Spalart-Allmaras model, this mesh is not adapted to capture such a partial cavity. With the other meshes, results are relatively similar at station 3,4 and 5. At station 2, as previously observed with the Smith model, the mesh $251 \times 77$ is near to provide a recirculating flow.

Figure 17 illustrates the velocity profiles for the experiments and the computations performed with the Menter SST model. At station 2, the re-entrant jet simulated by the mesh $251 \times 62$ is too extended. At station 3 , the intensity of the recirculating bubble varies with the mesh. With the coarsest mesh $251 \times 59$, the re-entrant jet is not predicted. It is under-predicted by the mesh $251 \times 61$ and well computed by the mesh $251 \times 62$. At stations 4 and 5 , results are quite similar. The Menter SST model seems rather sensitive to the near-wall mesh refinement with regard to the re-entrant jet development.

\subsection{Comparison of wall treatments}

The proposed TBLE wall model was validated on flat-plate turbulent boundarylayer flows [46].

For the cavitating simulations, only the coarse mesh $251 \times 62$ is used due to the good results obtained. Computations are performed with the two-layer wall functions and the TBLE model, associated with the Smith $k-l$ turbulence model. 
All global unsteady results are summarized in Table (3). The cavitation parameter in the inlet section $\sigma_{\text {inlet }}$ and the time-averaged cavity length obtained with both meshes are similar.

Figures 18 and 19 plot the velocity and void ratio profiles for the experiments and the computations from station 2 to 5, respectively. For all stations, results obtained with both wall treatments are similar and in very good agreement with the experimental data. For this quasi stable partial cavity, when the Smith model is used, the two-layer single-phase wall functions seem to be a good approximation.

\section{Computational results for a periodic self-oscillating cavity}

\subsection{Experimental conditions [47]}

This Venturi is characterized by a divergence angle of $8^{\circ}$, illustrated in Fig. 20. The geometrical data are:

Inlet section: $S_{i}=50 \times 44 \mathrm{~mm}^{2}$ (where the reference pressure is measured);

Throat section: $S_{\text {throat }}=33.5 \times 44 \mathrm{~mm}^{2}$;

Length of the test section (chord): $L_{r e f}=224 \mathrm{~mm}$.

This geometry is equipped with three probing holes to take various measurements such as the local void ratio and instantaneous local velocity. The selected operating point is characterized by the following physical parameters [47]:

$U_{\text {inlet }}=7.04 \mathrm{~m} / \mathrm{s}:$ the inlet velocity

$P_{\text {inlet }}=55000 \mathrm{~Pa}$ : the pressure in the inlet section 
$\sigma_{\text {inlet }}=\frac{P_{\text {inlet }}-P_{\text {vap }}}{0.5 \rho U_{\text {inlet }}^{2}} \simeq 2.15 \pm 0.06$ : the cavitation parameter in the inlet section

$T_{\text {ref }} \simeq 293 K$ : the reference temperature

$R e_{L_{r e f}}=\frac{U_{\text {inlet }} L_{r e f}}{\nu}=1.5710^{6}:$ the Reynolds number

With these parameters, an unsteady cavity with quasi-periodic fluctuations of the attached sheet and vapour cloud shedding has been obtained. Fig. 21 shows an instantaneous photograph of the cavity with a large structure shedding. The maximum length $L$ of the attached cavity is about $45 \mathrm{~mm}$. The vapour shedding frequency is close to $45 \mathrm{~Hz}$ estimated from Fourier transformations of unsteady pressure signals.

\subsection{Meshes}

The initial grid is a H-type topology. It contains 174 nodes in the flow direction and 62 in the orthogonal direction. A special contraction of the mesh is applied in the main flow direction just after the throat to better simulate the two-phase flow area (Fig. 22). The $y^{+}$values of the mesh, at the center of the first cell, vary between 2 and 6 for a non cavitating computation. A similar grid was used successfully for such cavity simulations in [47].

From this grid, five meshes are built by adding or eliminating cells near the wall. A view of all meshes near the throat is illustrated in Fig. 23. The $y^{+}$ values are presented in Fig. 24 obtained from a non cavitating computation with the Spalart-Allmaras turbulence model. Simulations performed with the finest mesh $174 \times 77$ do not use wall functions. 


\subsection{Numerical parameters}

This case is stiffer that the previous partial cavity. All unsteady computations were performed with the same value of the dimensionless time step: $\Delta t^{*}=\frac{\Delta t U_{\text {inlet }}}{L_{\text {ref }}}=6.410^{-4}$, and the same number of sub-iterations for the dual time method: 100 .

\subsection{Mesh influence}

\subsection{1. $C P U$ cost}

The CPU cost is presented in Table 4 for all meshes using the SpalartAllmaras turbulence model. Computations were performed on a Xeon Altix XE240 server with 4 processors. The ratio between CPU time for a given case and the fine mesh computation is also given. With the coarsest mesh, the gain is around $25 \%$.

\subsubsection{Global analyses}

Only the Spalart-Allmaras turbulence model is used. The CPU cost of a complete study with all turbulence models is too high. All calculations were performed by considering different sigma inlet values, summarized in Table (5). The goal was to obtain a periodic sheet cavity with a frequency close to $45 \mathrm{~Hz}$. The simulation time is around $2 \mathrm{~s}$. For all cases, a direct Fourier transformation (DFT) of the vapour volume signal was performed to evaluate the frequency.

Firstly, with the finest mesh $174 \times 77$ computed without wall functions, it was not possible to capture a periodic self-oscillating cavity with a frequency close to $45 \mathrm{~Hz}$. For values of the inlet cavitation parameter near 2.15, the 
obtained frequency is around $30 \mathrm{~Hz}$. For higher values of $\sigma_{\text {inlet }}$, no frequency appears (see Figure 25).

For all other meshes computed with wall functions, we can see two types of behaviour according to the mesh refinement. For the "finer" meshes $174 \times 62$ and $174 \times 60$, the frequency is around $35 \mathrm{~Hz}$ when the cavitation parameter is close to the experimental value. When this value is increased, the frequency increases and exceeds $40 \mathrm{~Hz}$. We can suppose that, for higher values of $\sigma_{\text {inlet }}$, the computed frequency could reach $45 \mathrm{~Hz}$. Besides, with the coarsest meshes, both the frequency and the $\sigma_{\text {inlet }}$ value are close to the experimental data. Figure 25 (down) presents the DFT of the vapour volume obtained with the mesh $174 \times 57$.

\subsubsection{Velocity and void ratio profiles}

The experimental data are obtained by a double optical probe [47]. Identically to the previous case, the velocity is evaluated as the most probable value and the void ratio is obtained with a post-processing algorithm. For the velocity profiles, experimental data are given for the first two stations. All numerical values were obtained by a time-averaged treatment.

Figure 26 shows the evolution of the longitudinal velocity for the experiments and computations corresponding to the cases 1, 4, 6, 7, 8 and 9 (see Table 5) where the frequency is the closest to the experimental value. All cases provided similar results. The re-entrant jet phenomenon is well captured with all meshes.

Figure 27 illustrates experimental and numerical results concerning the void ratio profiles. Experimental values are weak, even at station 1 near the 
throat. With the three coarser meshes $174 \times 59,174 \times 57$ and $174 \times 56$ (left), the void radio at station 1 is largely over-predicted, up to a factor of 4 . At station 2 , on the contrary, the void ratio is under-predicted.

With the three finer meshes (right), the void ratio is less important at station 1 , due to the higher value of $\sigma_{\text {inlet }}$. At station 2 , the void ratio is close to zero, cavities being too small.

At station 3, all computations simulate a pure liquid profile (not presented). All meshes provide a small attached cavity, and downstream a fluctuating recirculation with two-phase structures shedding. These shedding are not extended and are rapidly eliminated. In comparison with the experimental visualizations, the attached cavity and the clouds shedding are largely underestimated.

\subsubsection{Wall pressure distribution}

The time-averaged dimensionless wall pressure distribution is plotted in Fig. 28 versus the distance $x-x_{\text {inlet }}$. Results obtained with all meshes are similar, in very close agreement to the experimental data. The mesh influence is very weak with regard to the wall pressure evolution.

\subsection{Comparison of wall treatments}

For this study, only the coarsest mesh $174 \times 56$ is used. Computations are performed with the two-layer wall functions and the TBLE model associated with the $k-l$ turbulence model.

The CPU cost ratio is around 1.12 in comparison with the standard wall law simulation. 
All global unsteady results are summarized in Table (6). With both wall models, a periodic cycle around $45 \mathrm{~Hz}$ is computed, and the cavitation parameter in the inlet section $\sigma_{\text {inlet }}$ is close to the experimental value.

Figure 29 illustrates the velocity profiles for the experiment and the computations, at station 1 and 2. Results are similar.

The void ratio profiles are plotted in Fig. 30 for the experiment and the computations. At station 1, profiles provided by both wall models are identical. At station 2, the profile is over-predicted by the TBLE model and a little under-predicted by the standard wall functions. At station 3, all computations provided a pure liquid profile (not presented). The TBLE model does not allow us to obtain a more extended cloud shedding.

The time-averaged dimensionless wall pressure distribution is shown in Fig. 31 versus the distance $x-x_{\text {inlet }}$. As previously, results are quite similar.

For this unstable partial cavity, when the Smith model is used, the two-layer single-phase wall model seems to be a good approximation, as previously concluded for the quasi stable case.

\section{Summary and conclusions}

The simulation of partial cavities appearing along solid boundaries is investigated with a special focus on the turbulent wall treatment and a mesh refinement near walls. A two-phase one-fluid compressible CFD solver was applied to simulate unsteady quasi stable and unstable cavity sheets. Various transport-equation turbulence models were tested and two wall functions were compared. The numerical results demonstrated the influence of the near-wall boundary layer modelling to capture unsteady behaviours of cavity 
sheets. Global and local analyses of flows were proposed based on void ratio and velocity profiles.

Firstly, a comparison of computations performed with and without wall functions was proposed. For the quasi-stable cavity, except the Menter model, the re-entrant jet simulated with the fine mesh was more extended in comparison with the wall function computations and the experimental data. With the Menter SST model, a singular result was exhibited with the fine mesh: the computation did not predict a significant re-entrant jet. For the periodic unstable cavity, only wall function computations were allowed to obtain a periodic cycle close to the experimental frequency. Fine mesh computations performed with the Spalart-Allmaras model were unable to predict the shedding frequency.

Secondly, results obtained with various coarse meshes for which the wall zone differs were presented. For the quasi-stable cavity, the sensitivity to the mesh refinement varies according to the considered turbulence model, with regard to the re-entrant jet development. The mesh influence was weak with the Smith model while it was pronounced with the Spalart-Allmaras model and the Menter model. For the unstable cavity, with regard to the frequency and $\sigma_{\text {inlet }}$ values, best results were obtained with the coarsest meshes. Yet, the local velocity profiles obtained with all meshes were similar. It is difficult to explain the better results obtained with coarse meshes, especially for the unstable cavity. Probably, the wall treatment allows to reduce some problems of the turbulence models due to the weakness of the wall damping functions.

Finally, a new wall treatment based on the local integration of the simplified 
boundary layer equations (TBLE model) was proposed and compared with the two-layer law of the wall. This wall model allows to account for the adverse pressure gradient in recirculating areas and to avoid assumptions about the existence of a universal mean-velocity profile. Even for single-phase flows, this aspect is controverted and the validity of the classical log-law is discussed. In two-phase cavitating flows, the validity of the log-law was never checked. The TBLE approach can be used for a posteriori validation in which the TBLE solution is considered as a reference solution.

For both quasi stable and unstable cavities, using the Smith turbulence model, first results demonstrated the good behaviour of the two-layer wall model. Indeed, results obtained are similar to those provided by the TBLE model. It is not necessary to include explicitly the pressure gradient in the mean-velocity profile to obtain correct results, even in a separated region.

Other computations by varying the embedded grid will be necessary to attest these results. Additional works are in progress to develop the TBLE model for other turbulence models, to investigate three-dimensional simulations, and to pursue comparative analyses between numerical and experimental studies. 
[1] K. Laberteaux, S. Ceccio, Partial cavity flows. Part1. Cavities forming on models without spanwise variation, Journal of Fluid Mechanics 431 (2001) 1-41.

[2] D. de Lange, G. Bruin, L. van Wijngaarden, On the mechanism of cloud cavitation - experiments and modelling, in: 2nd International Symposium on Cavitation CAV1994, Tokyo, Japan, 1994.

[3] Y. Kawanami, H. Kato, H. Yamaguchi, M. Tanimura, Y. Tagaya, Mechanism and control of cloud cavitation, Journal of Fluids Engineering 119 (4) (1997) 788-794.

[4] S. Gopalan, J. Katz, Flow structure and modeling issues in the closure region of attached cavitation, Physics of Fluids 12 (4) (2000) 895-911.

[5] V. Ahuja, A. Hosangadi, S. Arunajatesan, Simulations of cavitating flows using hybrid unstructured meshes, Journal of Fluids Engineering 123 (2) (2001) 331-340.

[6] O. Coutier-Delgosha, J. Reboud, Y. Delannoy, Numerical simulation of the unsteady behaviour of cavitating flow, Int. Journal for Numerical Methods in Fluids 42 (2003) 527-548.

[7] R. Kunz, D. Boger, D. Stinebring, T. Chyczewski, J. Lindau, H. Gibeling, S. Venkateswaran, T. Govindan, A preconditioned navier-stokes method for two-phase flows with application to cavitation prediction, Computers \& Fluids 29 (8) (2000) 849-875.

[8] J. Lindau, R. Kunz, D. Boger, D. Stinebring, H. Gibeling, High Reynolds 
number, unsteady, multiphase CFD modeling of cavitating flows, Journal of Fluids Engineering 124 (3) (2002) 124-607.

[9] I. Senocak, W. Shyy, A pressure-based method for turbulent cavitating flow computations, Journal of Computational Physics 176 (2) (2002) 363-383.

[10] A. Singhal, M. Athavale, H. Li, Y. Jiang, Mathematical basis and validation of the full cavitation model, Journal of Fluids Engineering 124 (3) (2002) 617-624.

[11] E. Sinibaldi, F. Beux, M. Salvetti, A numerical method for 3D barotropic flows in turbomachinery, Flow Turbulence Combustion 76 (2006) 371381.

[12] J. Wu, G. Wang, W. Shyy, Time-dependent turbulent cavitating flow computations with interfacial transport and filter-based models, Int. Journal for Numerical Methods in Fluids 49 (7) (2005) 739-761.

[13] C. Sarraf, Y. A. Bouziad, H. Djeridi, M. Farhat, F. Deniset, J.-Y. Billard, Effet of cavitation on the structure of the boundary layer in the wake of a partial cavity, in: $6^{\text {th }}$ International Symposium on Cavitation CAV2006, Wageningen, The Netherlands, 2006.

[14] E. Goncalves, R. F. Patella, Numerical simulation of cavitating flows with homogeneous models, Computers \& Fluids 38 (9) (2009) 16821696.

[15] E. Goncalves, R. F. Patella, Numerical study of cavitating flows with thermodynamic effect, Computers \& Fluids 39 (1) (2010) 99-113. 
[16] E. Goncalves, Numerical study of unsteady turbulent cavitating flows, European Journal of Mechanics B/Fluids 30 (1) (2010) 26-40.

[17] H. Guillard, C. Viozat, On the behaviour of upwind schemes in the low Mach number limit, Computers \& Fluids 28 (1) (1999) 63-86.

[18] E. Turkel, Preconditioned methods for solving the incompressible and low speed compressible equations, Journal of Computational Physics 172 (2) (1987) 277-298.

[19] C. Ishii, T. Hibiki, Thermo-fluid dynamics of two-phase flow, Springer (2006).

[20] Y. Delannoy, J. Kueny, Two phase flow approach in unsteady cavitation modelling, in: Cavitation and Multiphase Flow Forum, ASME-FED, vol. 98, pp.153-158, 1990.

[21] P. Spalart, S. Allmaras, A one-equation turbulence model for aerodynamic flows, in: AIAA 92-0439, 30 ${ }^{\text {th }}$ Aerospace Sciences Meeting Reno, Nevada, 1992.

[22] P. Spalart, S. Allmaras, A one-equation turbulence model for aerodynamic flows, La Recherche Aérospatiale (1) (1994) 5-21.

[23] B. Smith, A near wall model for the $k-l$ two equation turbulence model, in: AIAA 94-2386, 25 $5^{\text {sh }}$ Fluid Dynamics Conference - Colorado Springs, Colorado, 1994.

[24] B. Smith, The $k-k l$ turbulence model and wall layer model for com- 
pressible flows, in: AIAA 90-1483, $21^{\text {st }}$ Fluid and Plasma Dynamics Conference - Seattle, Washington, 1990.

[25] F. Menter, Zonal two equation $k-\omega$ turbulence models for aerodynamic flows, in: AIAA 93-2906, 24th Fluid Dynamics Conference - Orlando, Florida, 1993.

[26] F. Menter, Two-equation eddy-viscosity turbulence models for engineering applications, AIAA Journal 32 (8) (1994) 1598-1605.

[27] W. Jones, B. Launder, The prediction of laminarization with a twoequation model of turbulence, Int. J. Heat Mass Transfer 15 (1972) $301-314$.

[28] P. Huang, G. Coleman, P. Bradshaw, Compressible turbulent channel flows: DNS results and modelling, Journal of Fluid Mechanics 305 (1995) $185-218$.

[29] S. Sarkar, The stabilizing effect of compressibility in turbulent shear flow, Journal of Fluid Mechanics 282 (1995) 163-186.

[30] R. Lechner, J. Sesterhenn, R. Friedrich, Turbulent supersonic channel flow, Journal of Turbulence 2 (1).

[31] J.-L. Reboud, B. Stutz, O. Coutier, Two-phase flow structure of cavitation: experiment and modelling of unsteady effects, in: $3^{\text {rd }}$ International Symposium on Cavitation CAV1998, Grenoble, France, 1998.

[32] J. Viegas, M. Rubesin, Wall-function boundary conditions in the solution of the Navier-Stokes equations for complex compressible flows, in: 
AIAA 83-1694, $16^{\text {th }}$ Fluid and Plasma Dynamics Conference - Danver, Massachussetts, 1983.

[33] E. Goncalves, R. Houdeville, Reassessment of the wall functions approach for RANS computations, Aerospace Science and Technology 5 (2001) 1-14.

[34] U. Piomelli, Wall-layer models for large-eddy simulations, Progress in Aerospace Sciences 44 (2008) 437-446.

[35] M. Buschmann, M. G. el Hak, Recent developments in scaling of wallbounded flows, Progress in Aerospace Sciences 42 (2007) 419-467.

[36] A. Smits, B. McKeon, I. Marusic, High-reynolds number wall turbulence, Annual Review of Fluid Mechanics 43 (2011) 353-375.

[37] E. Balaras, C. Benocci, U. Piomelli, Two-layer approximate boundary conditions for large-eddy simulation, AIAA Journal 34 (1996) 11111119.

[38] W. Cabot, P. Moin, Approximate wall boundary conditions in the largeeddy simulation of high Reynolds number flow, Flow, Turbulence and Combustion 63 (1999) 269-291.

[39] M. Wang, P. Moin, Dynamic wall modeling for large-eddy simulation of complex turbulent flows, Physic of Fluids 14 (7) (2002) 2043-2051.

[40] A. Jameson, W. Schmidt, E. Turkel, Numerical solution of the Euler equations by finite volume methods using Runge-Kutta time stepping schemes, in: AIAA Paper 81-1259, 1981. 
[41] P. Roe, Approximate Riemann solvers, parameters vectors, and difference schemes, Journal of Computational Physics 43 (1981) 357-372.

[42] S. Tatsumi, L. Martinelli, A. Jameson, Flux-limited schemes for the compressible Navier-Stokes equations, AIAA Journal 33 (2) (1995) 252261.

[43] H. Luo, J. Baum, R. Lohner, A fast, matrix-free implicit method for compressible flows on unstructured grids, Journal of Computational Physics 146 (2) (1998) 664-690.

[44] B. Merci, J. Steelant, J. Vierendeels, K. Riemslagh, E. Dick, Computational treatment of source terms in two-equation turbulence models, AIAA Journal 38 (11) (2000) 2085-2093.

[45] S. Barre, J. Rolland, G. Boitel, E. Goncalves, R. F. Patella, Experiments and modelling of cavitating flows in Venturi: attached sheet cavitation, European Journal of Mechanics B/Fluids 28 (2009) 444-464.

[46] E. Goncalves, Implantation et validation de lois de paroi dans un code navier-stokes, Ph.D. thesis, Ecole Nationale Superieure de l'Aeronautique et de l'Espace (2001).

[47] R. F. Patella, S. Barre, J. Reboud, Experiments and modelling of cavitating flows in Venturi : part II, unsteady cavitation, in: 6th International Symposium on Cavitation CAV2006, Wageningen, The Netherlands, 2006. 
Table 1: Comparison with and without wall functions, $4^{\circ}$ Venturi.

\begin{tabular}{lcccl}
\hline$\sigma_{\text {inlet }}(\operatorname{exp~0.55)}$ & $\mathrm{SA}$ & $\mathrm{KL}$ & $\mathrm{KE}$ & $\mathrm{KWSST}$ \\
\hline fine $251 \times 81$ & 0.62 & 0.605 & 0.60 & 0.665 \\
coarse $251 \times 62$ & 0.595 & 0.59 & 0.58 & 0.62 \\
& & & & \\
\hline$L_{\text {cav }}(\mathrm{mm})(\exp 70-85)$ & $\mathrm{SA}$ & $\mathrm{KL}$ & $\mathrm{KE}$ & $\mathrm{KWSST}$ \\
\hline fine $251 \times 81$ & 85 & 84 & 84 & 82 \\
coarse $251 \times 62$ & 80 & 76 & 83 & 79 \\
& & & & \\
\hline
\end{tabular}


Table 2: Mesh influence with wall functions, $4^{\circ}$ Venturi.

\begin{tabular}{lcccl}
\hline \hline$\sigma_{\text {inlet }}(\operatorname{exp~0.55)}$ & $\mathrm{SA}$ & $\mathrm{KL}$ & $\mathrm{KE}$ & $\mathrm{KWSST}$ \\
\hline $251 \times 77$ & 0.59 & 0.59 & 0.64 & 0.64 \\
$251 \times 62$ & 0.595 & 0.59 & 0.58 & 0.62 \\
$251 \times 61$ & 0.58 & 0.58 & 0.62 & 0.61 \\
$251 \times 59$ & 0.56 & 0.565 & 0.59 & 0.57 \\
& & & & \\
\hline$L_{\text {cav }}(\mathrm{mm})(\exp 70-85)$ & $\mathrm{SA}$ & $\mathrm{KL}$ & $\mathrm{KE}$ & $\mathrm{KWSST}$ \\
\hline $251 \times 77$ & 85 & 75 & 82 & 75 \\
$251 \times 62$ & 80 & 76 & 82 & 79 \\
$251 \times 61$ & 81 & 75 & 79 & 82 \\
$251 \times 59$ & 80 & 79 & 85 & 81 \\
& & & & \\
\hline
\end{tabular}


Table 3: Standard wall functions versus TBLE model, $4^{\circ}$ Venturi.

\begin{tabular}{lll}
\hline wall model & $\sigma_{\text {inlet }}$ & $L_{\text {cav }}(\mathrm{mm})$ \\
\hline standard wall law & 0.59 & 76 \\
TBLE & 0.587 & 76 \\
\hline
\end{tabular}


Table 4: CPU cost, Spalart-Allmaras model, $8^{\circ}$ Venturi.

\begin{tabular}{ccc}
\hline mesh & cost for 100 iterations (s) & ratio \\
\hline $174 \times 77$ & 525.6 & 1 \\
$174 \times 62$ & 429.6 & 0.817 \\
$174 \times 60$ & 419.4 & 0.80 \\
$174 \times 59$ & 408 & 0.776 \\
$174 \times 57$ & 393 & 0.747 \\
$174 \times 56$ & 387 & 0.736 \\
\hline
\end{tabular}


Table 5: Mesh influence, $8^{\circ}$ Venturi.

\begin{tabular}{lccc}
\hline case & mesh & $\sigma_{\text {inlet }}(\exp 2.15)$ & frequency $(\mathrm{Hz})(\exp 45)$ \\
\hline 1 & $174 \times 77$ & 2.13 & 30 \\
2 & & 2.18 & no frequency \\
\hline 3 & $174 \times 62$ & 2.13 & 35 \\
4 & & 2.17 & 40 \\
\hline 5 & $174 \times 60$ & 2.13 & 35 \\
6 & & 2.19 & 43.5 \\
\hline 7 & $174 \times 59$ & 2.14 & 44 \\
8 & $174 \times 57$ & 2.145 & 46 \\
9 & $174 \times 56$ & 2.14 & 46 \\
\hline
\end{tabular}


Table 6: Standard wall functions versus TBLE model, $8^{\circ}$ Venturi.

\begin{tabular}{lll}
\hline wall model & $\sigma_{\text {inlet }}$ & frequency $(\mathrm{Hz})$ \\
\hline standard wall law & 2.14 & 46 \\
TBLE & 2.10 & 43 \\
\hline
\end{tabular}




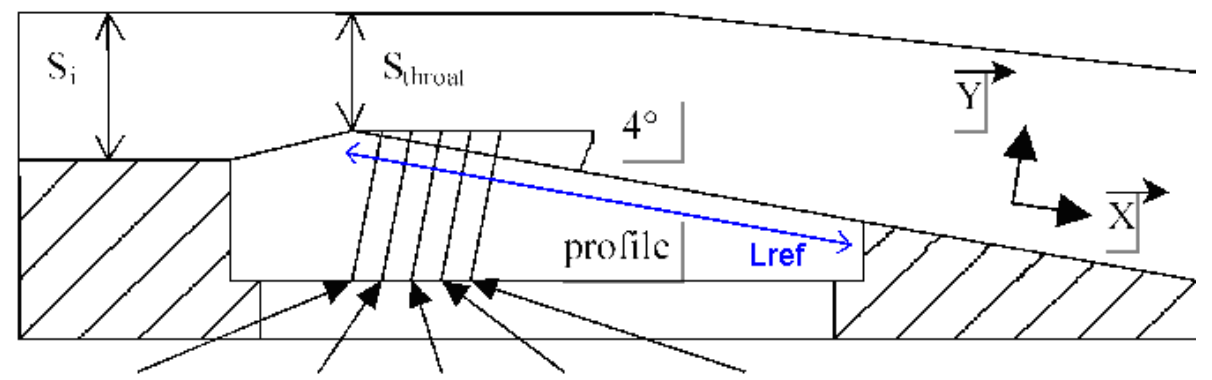

Station 1 Station 2 Station 3 Station 4 Station 5

Figure 1: Schematic view of the $4^{\circ}$ Venturi profile. 


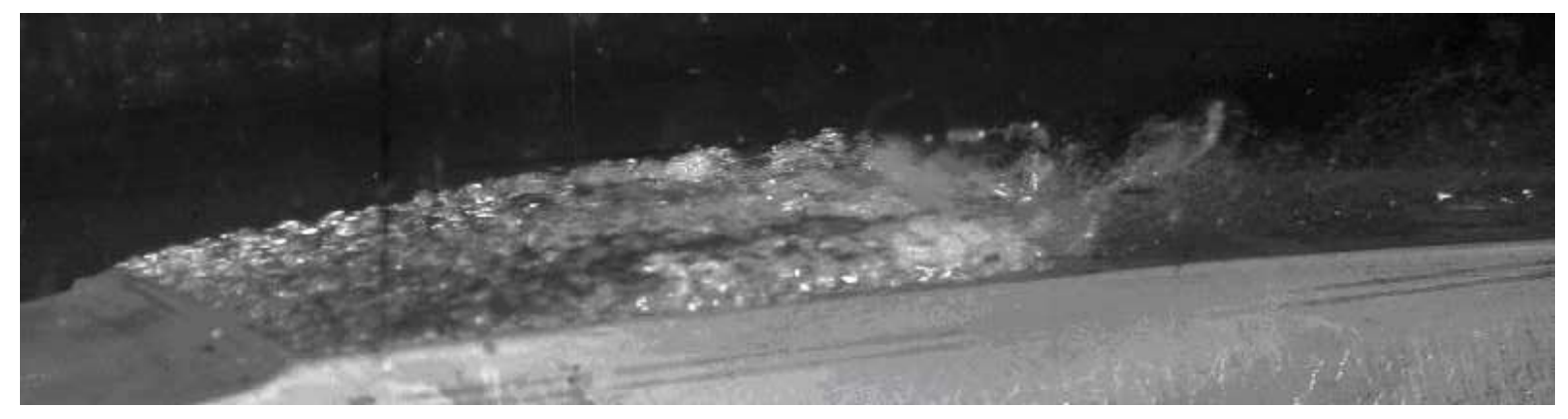

Figure 2: Photograph of the cavity, $4^{\circ}$ Venturi. 


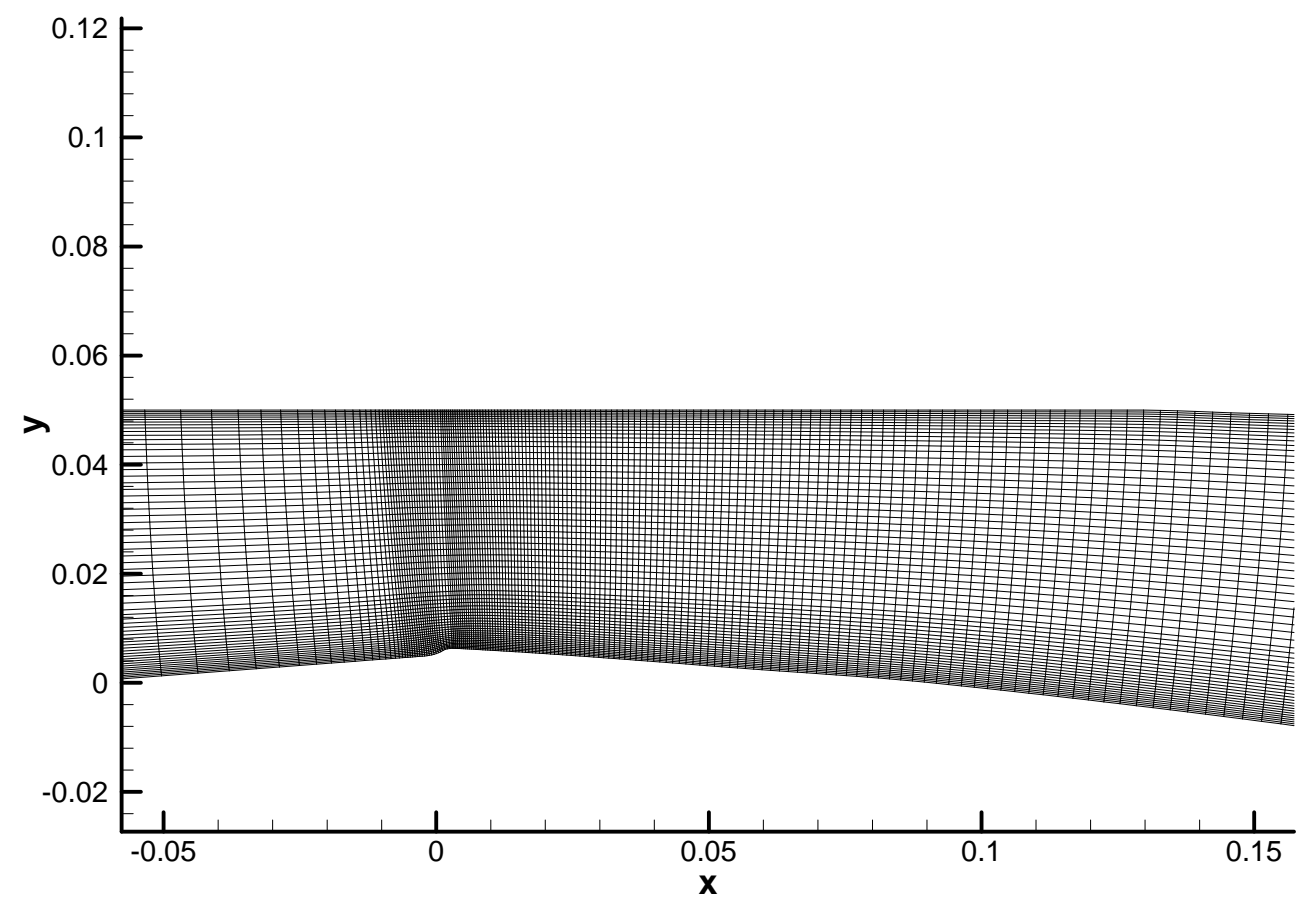

Figure 3: View of the mesh near the throat, $4^{\circ}$ Venturi. 

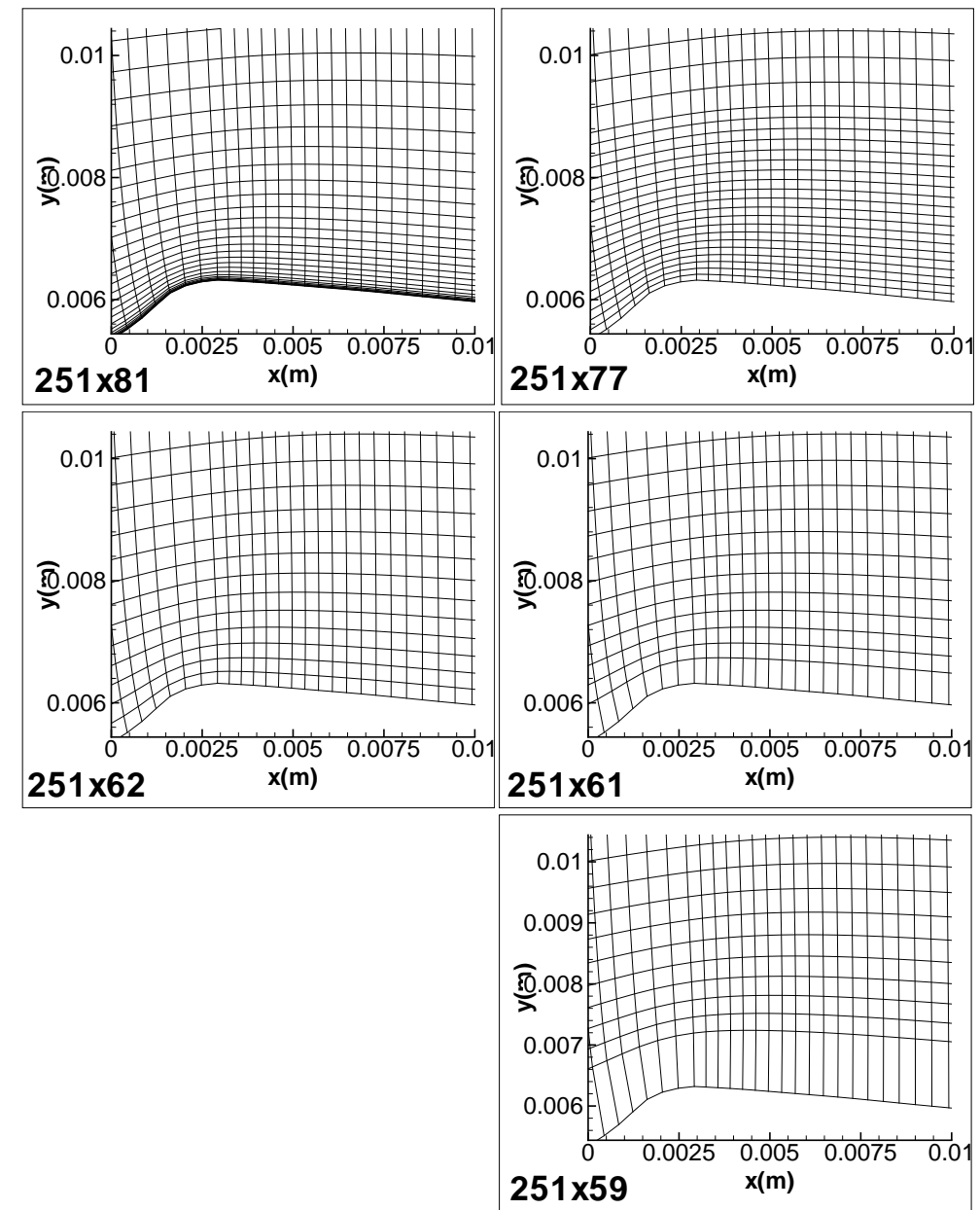

Figure 4: Enlargement of all meshes near the throat, $4^{\circ}$ Venturi. 


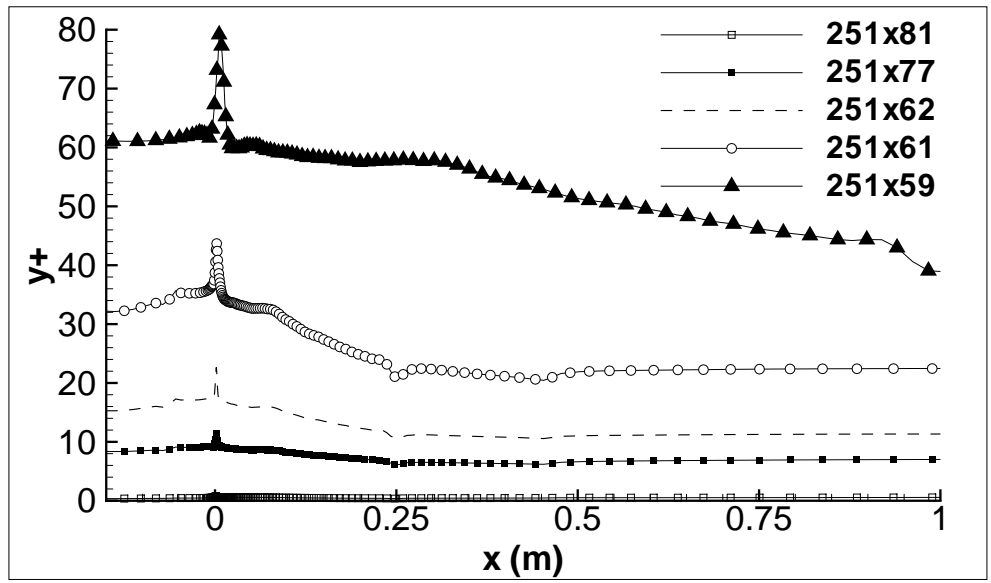

Figure 5: Evolution of the $y^{+}$values near the throat, $4^{\circ}$ Venturi. 

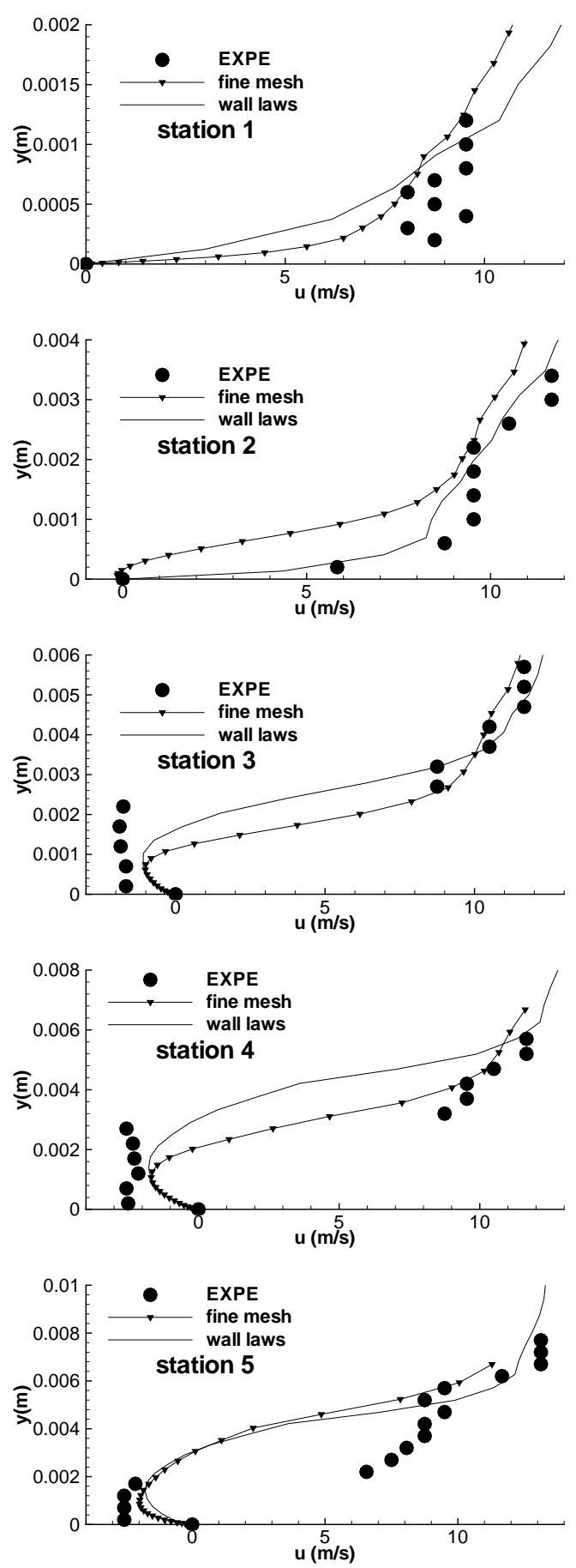

Figure 6: Time-averaged velocity profiles from station 1 to 5 , Spalart-Allmaras model, $4^{\circ}$ Venturi. 

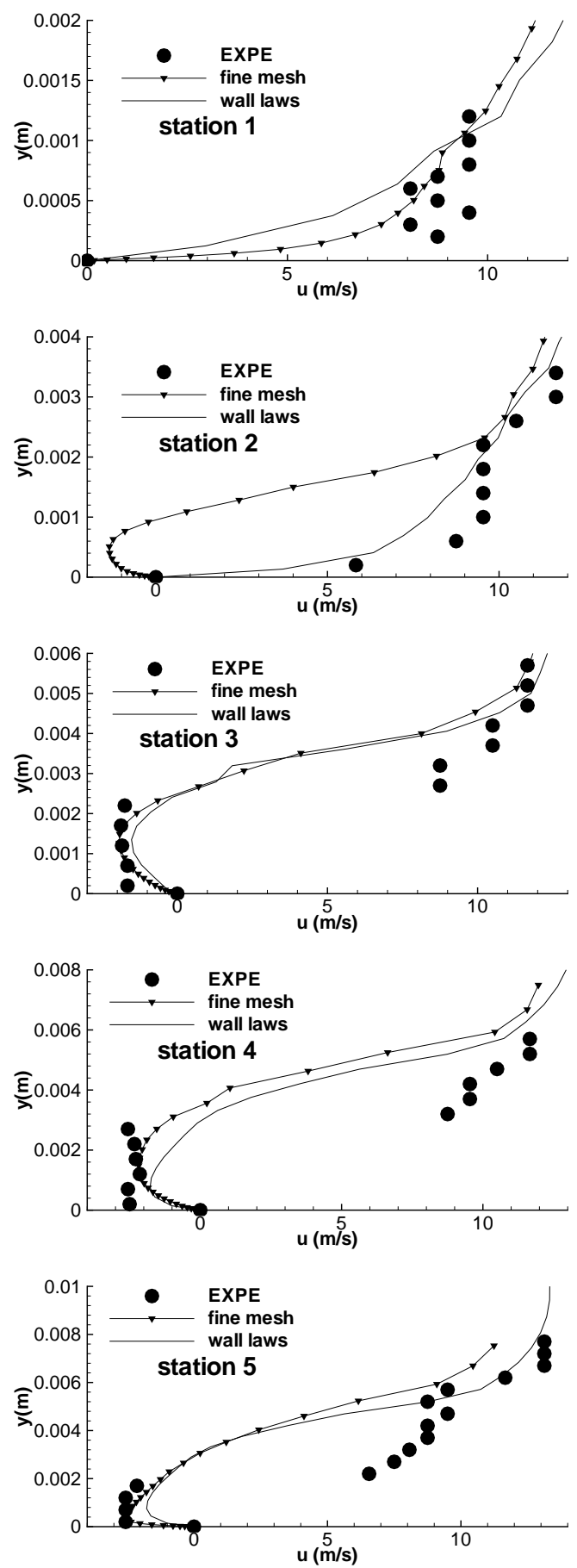

Figure 7: Time-averaged velocity profiles from station 1 to 5 , Smith $k-l$ model, $4^{\circ}$ Venturi. 

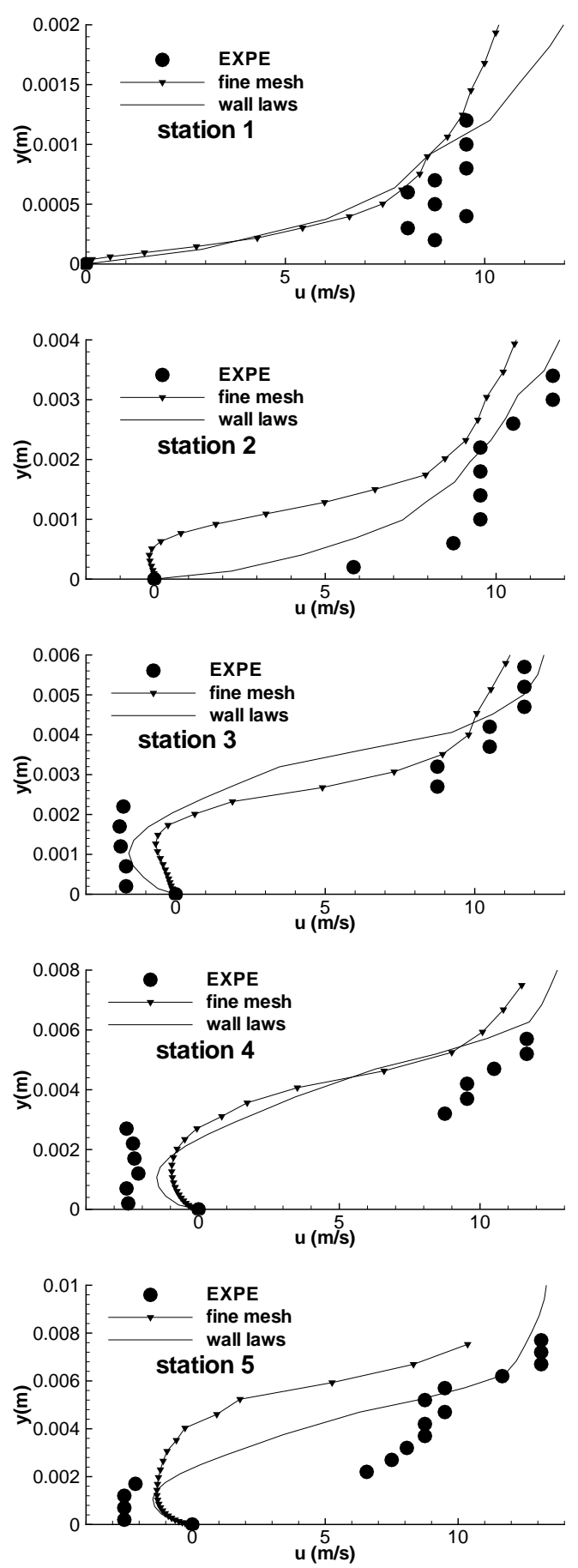

Figure 8: Time-averaged velocity profiles from station 1 to 5 , Jones-Launder $k-\varepsilon$ model, $4^{\circ}$ Venturi. 

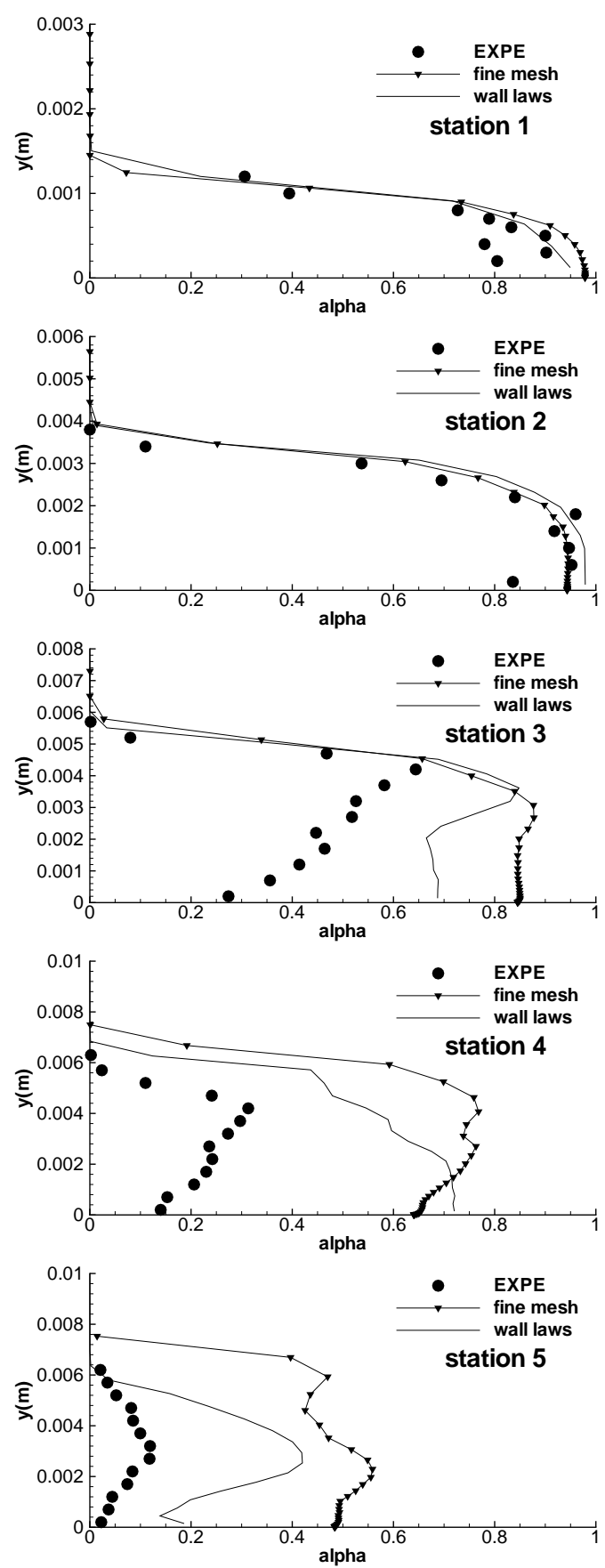

Figure 9: Time-averaged void ratio profiles from station 1 to 5, Spalart-Allmaras model, $4^{\circ}$ Venturi. 

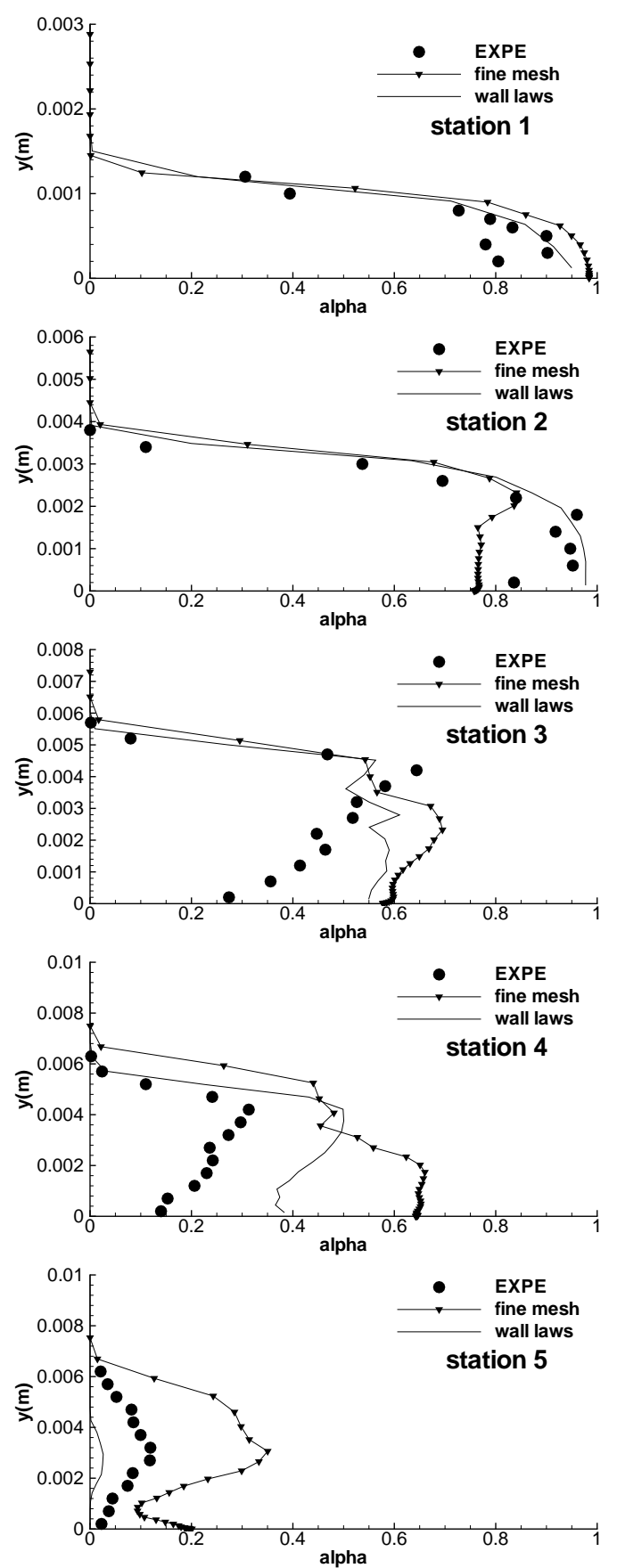

Figure 10: Time-averaged void ratio profiles from station 1 to 5 , Smith $k-l$ model, $4^{\circ}$ Venturi. 

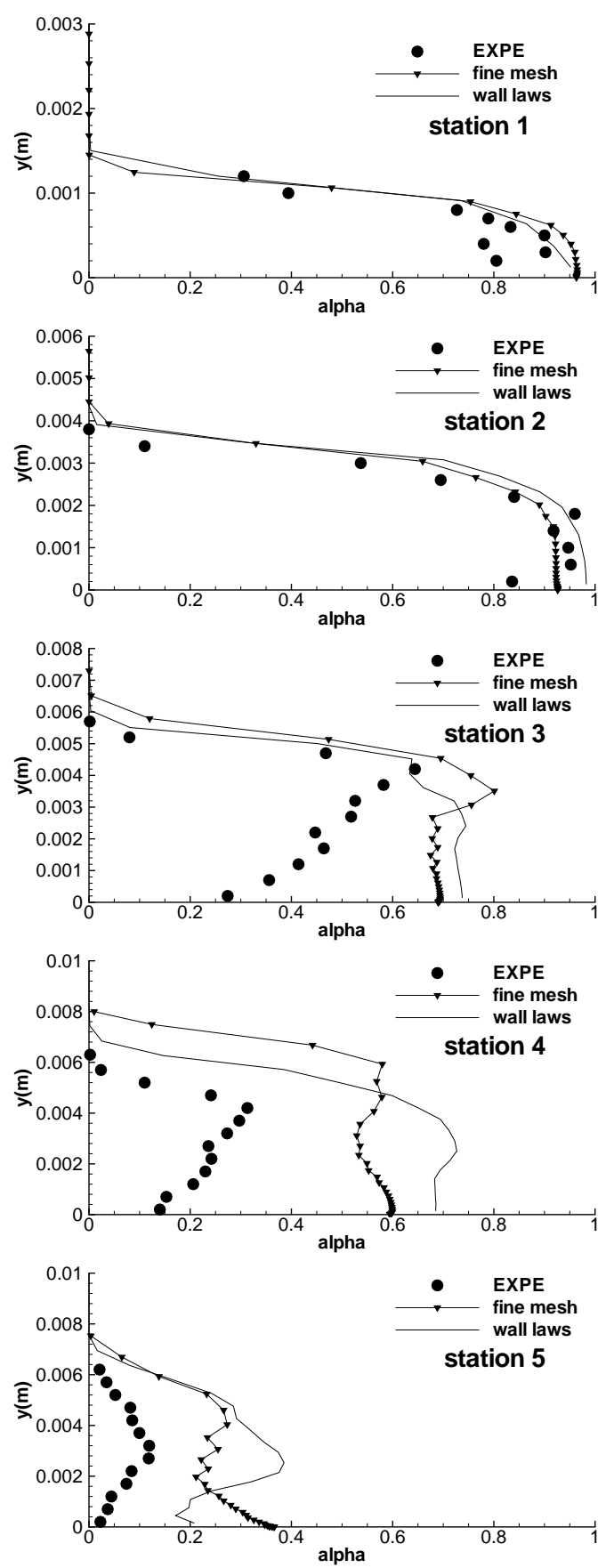

Figure 11: Time-averaged void ratio profiles from station 1 to 5 , Jones-Launder $k-\varepsilon$ model, $4^{\circ}$ Venturi. 

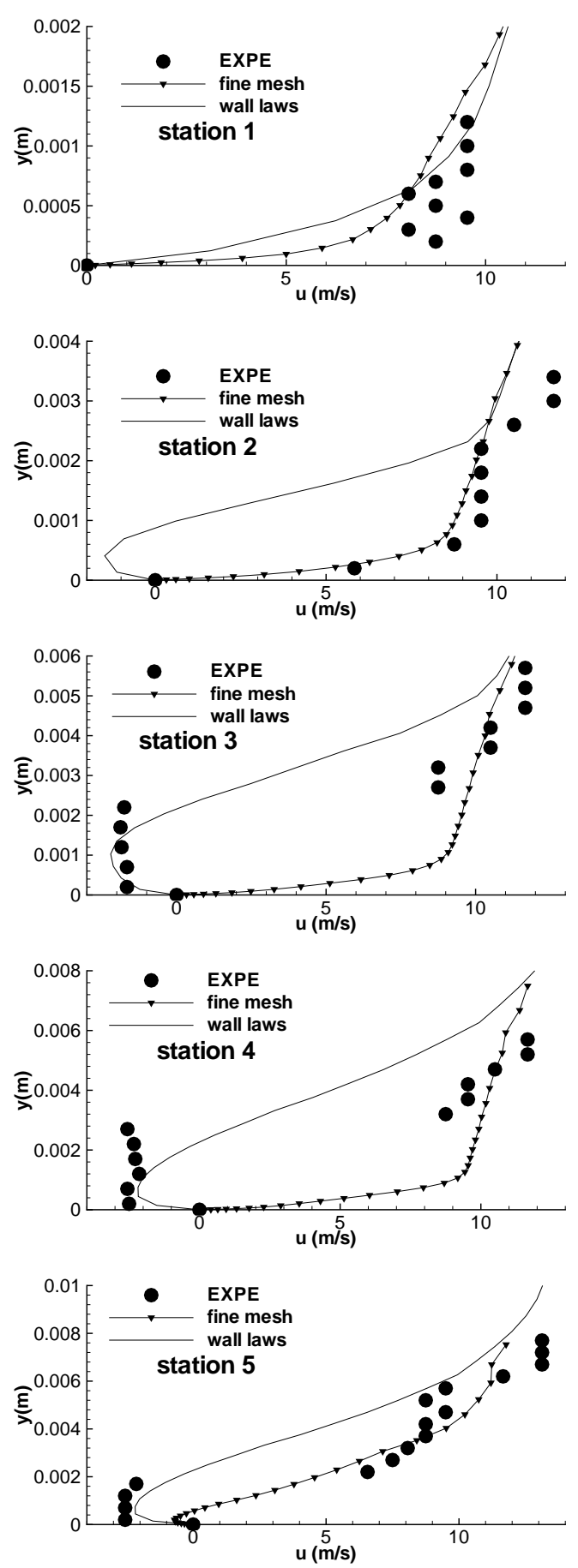

Figure 12: Time-averaged velocity profiles from station 1 to 5 , Menter SST model, $4^{\circ}$ Venturi. 

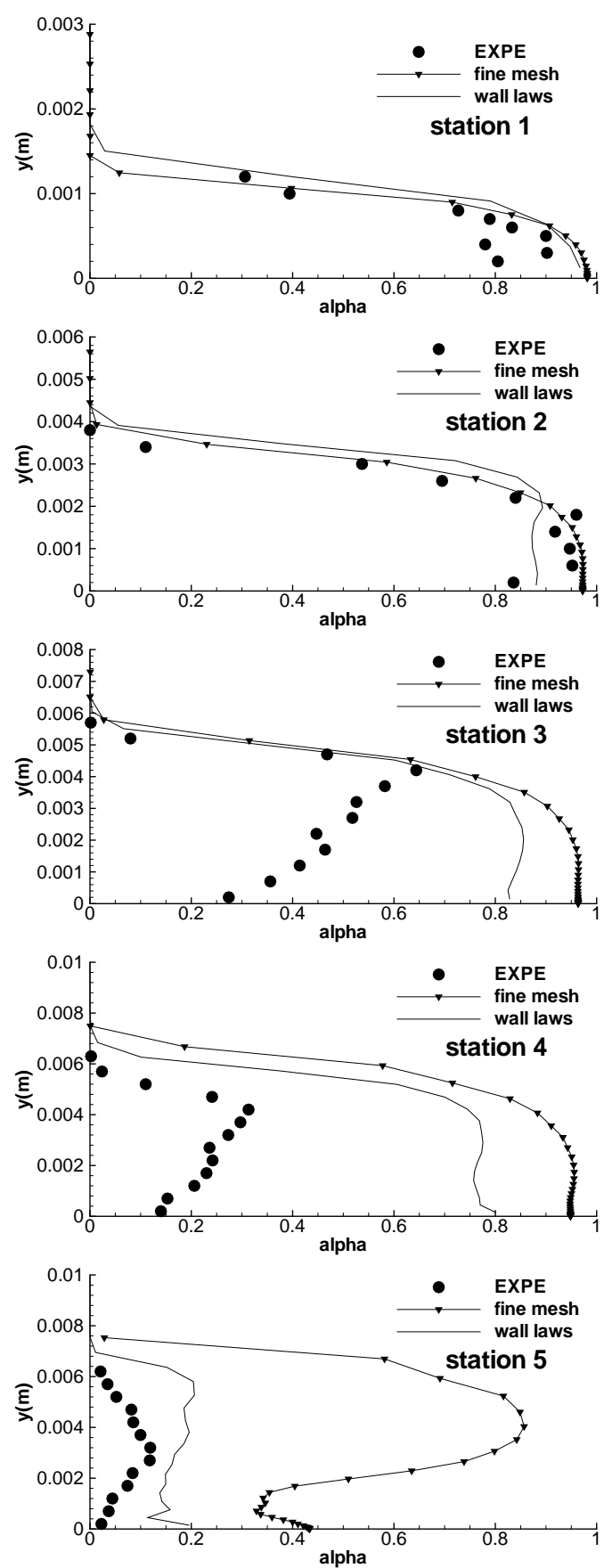

Figure 13: Time-averaged void ratio profiles from station 1 to 5 , Menter SST model, $4^{\circ}$ Venturi. 

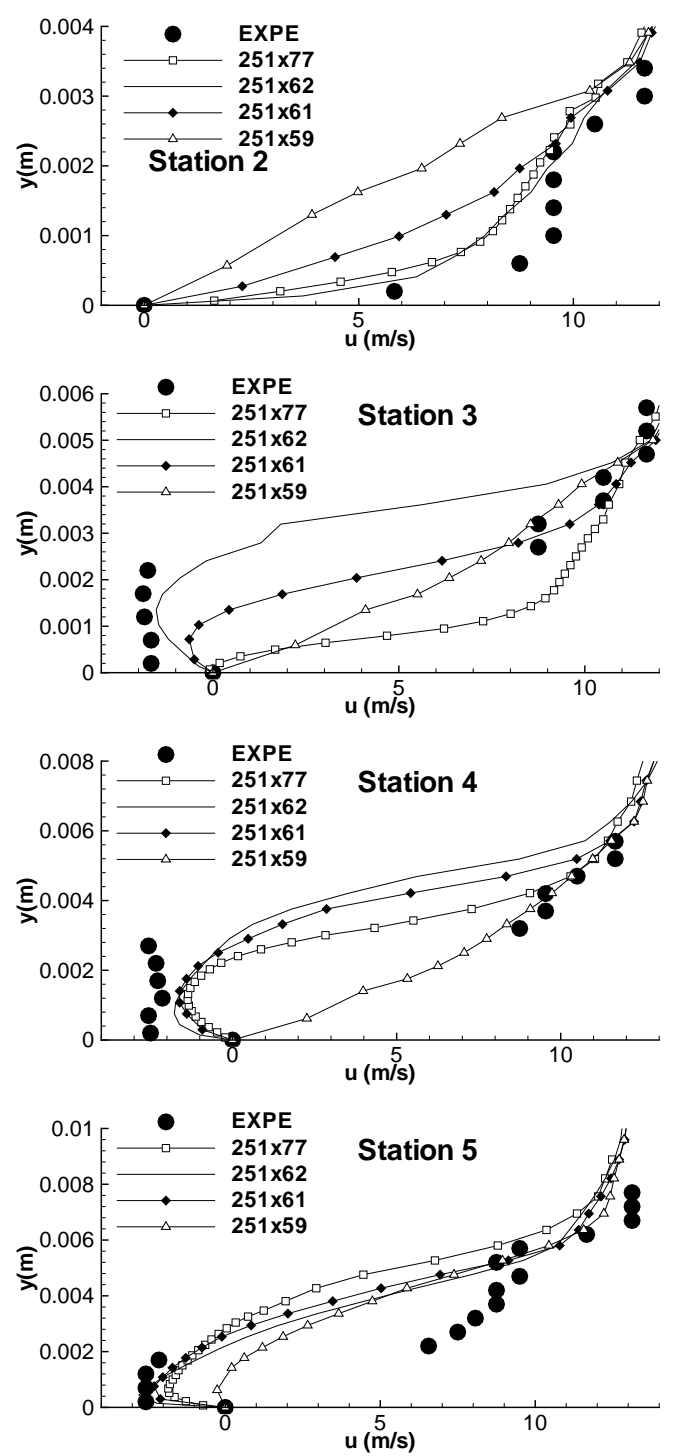

Figure 14: Time-averaged velocity profiles from station 2 to 5, Spalart-Allmaras model, $4^{\circ}$ Venturi. 

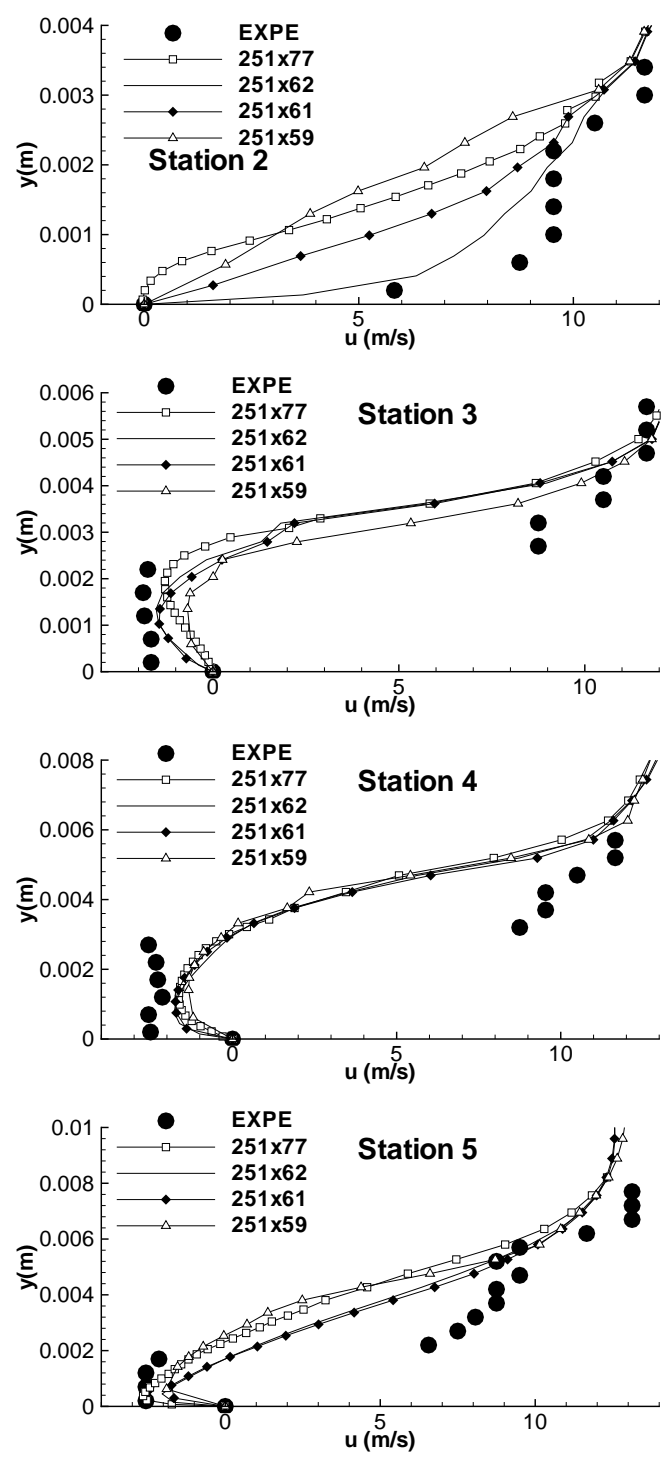

Figure 15: Time-averaged velocity profiles from station 2 to 5 , Smith $k-l$ model, $4^{\circ}$ Venturi. 

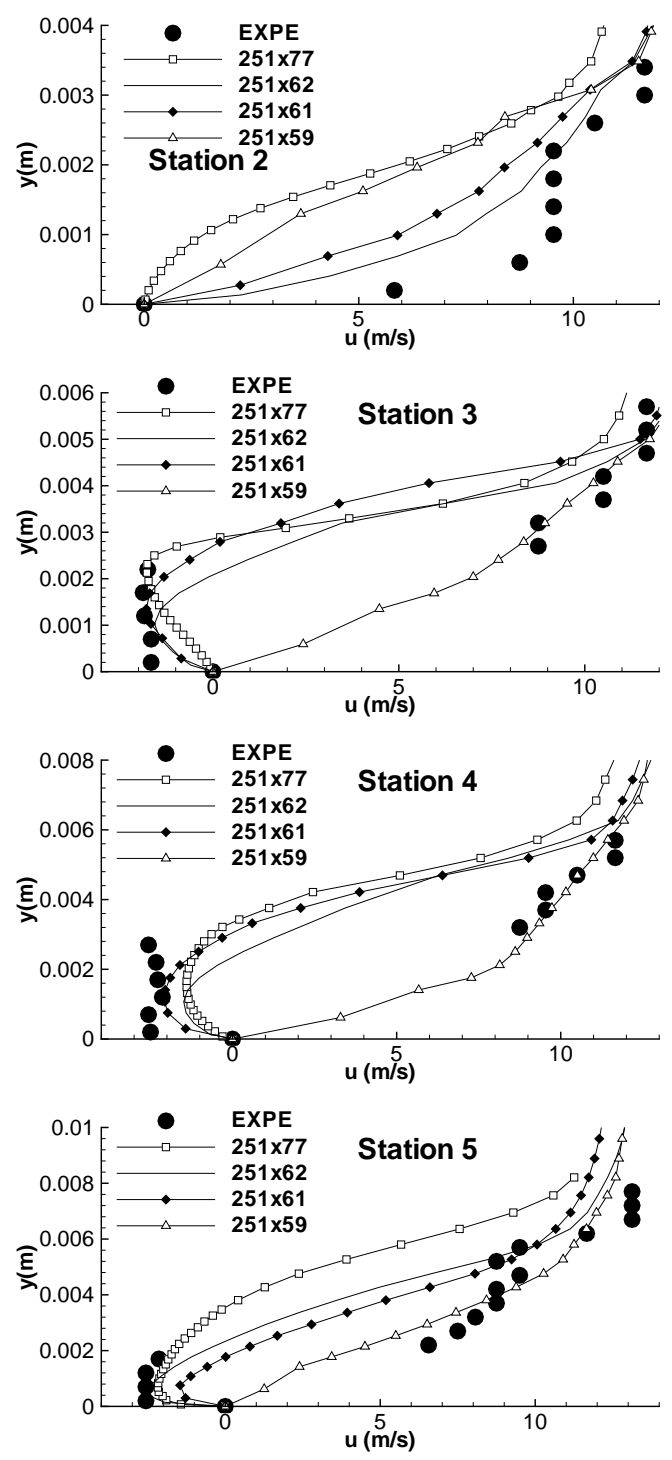

Figure 16: Time-averaged velocity profiles from station 2 to 5 , Jones-Launder $k-\varepsilon$ model, $4^{\circ}$ Venturi. 

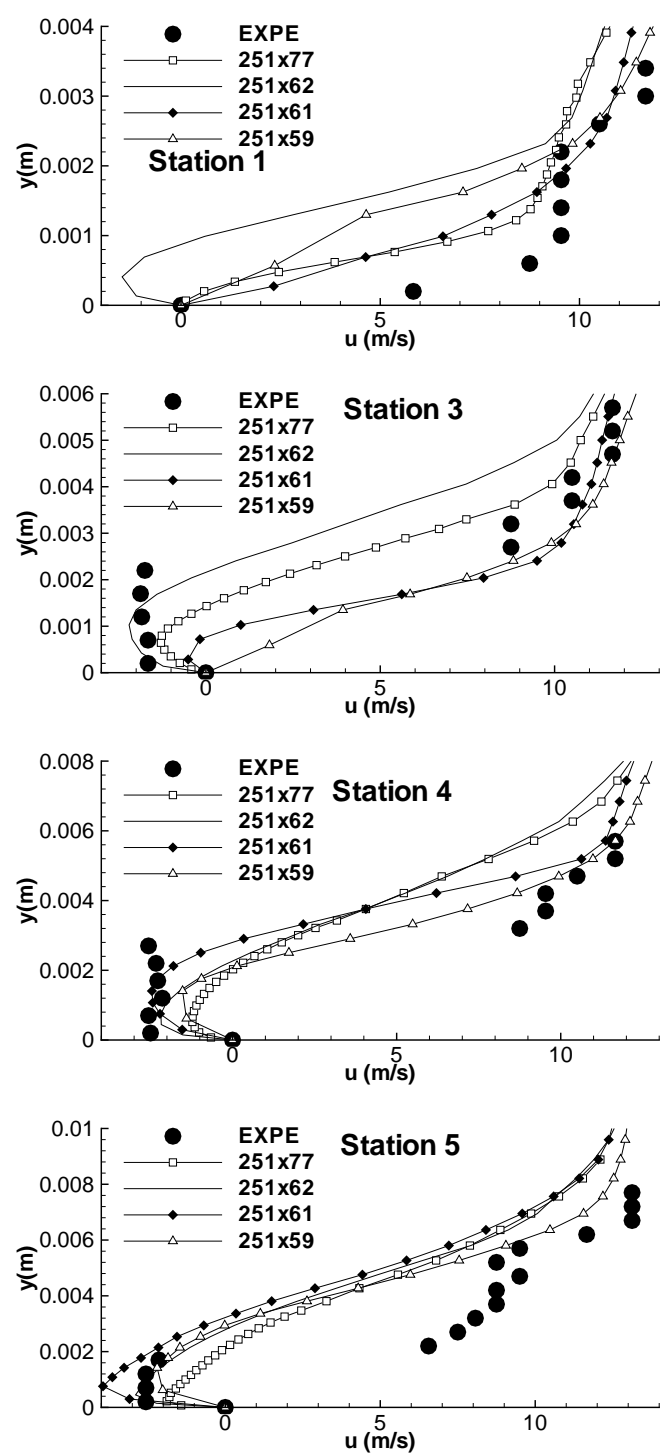

Figure 17: Time-averaged velocity profiles from station 2 to 5 , Menter $k-\omega$ SST model, $4^{\circ}$ Venturi. 

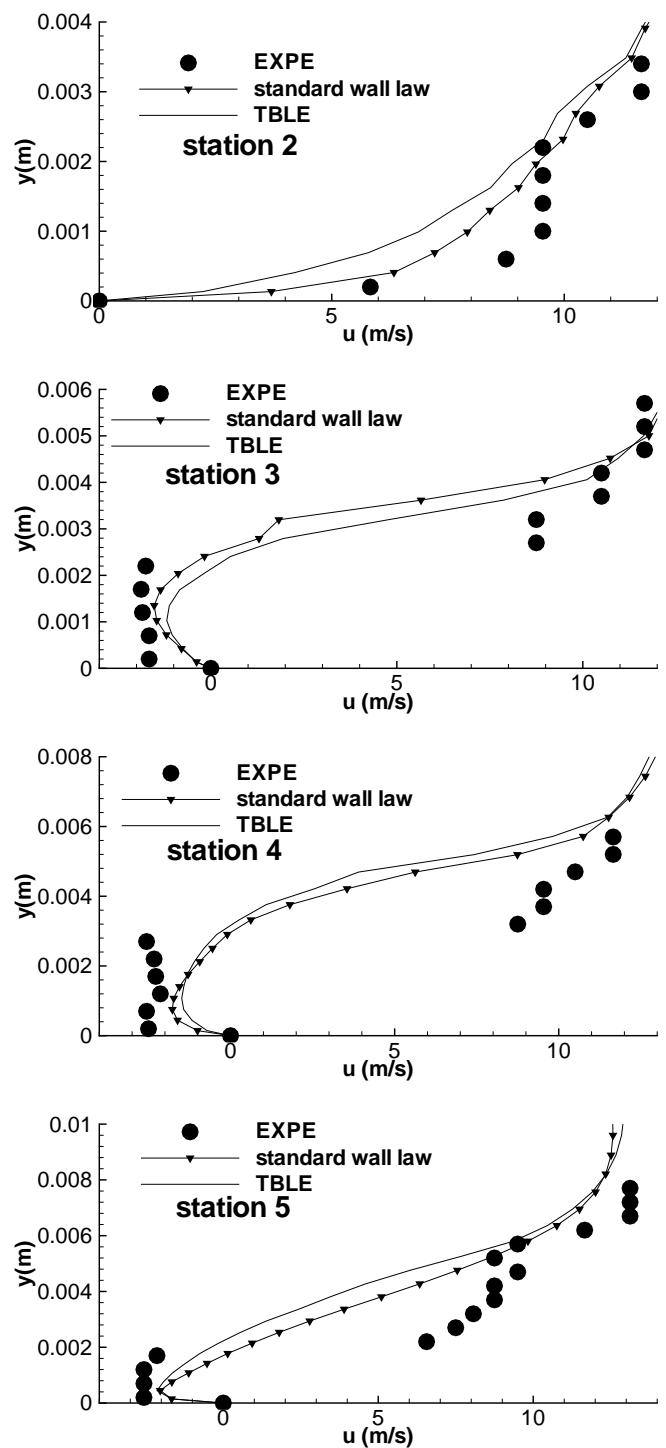

Figure 18: Time-averaged velocity profiles from station 2 to 5 , Smith $k-l$ model, TBLE versus standard wall law, $4^{\circ}$ Venturi. 

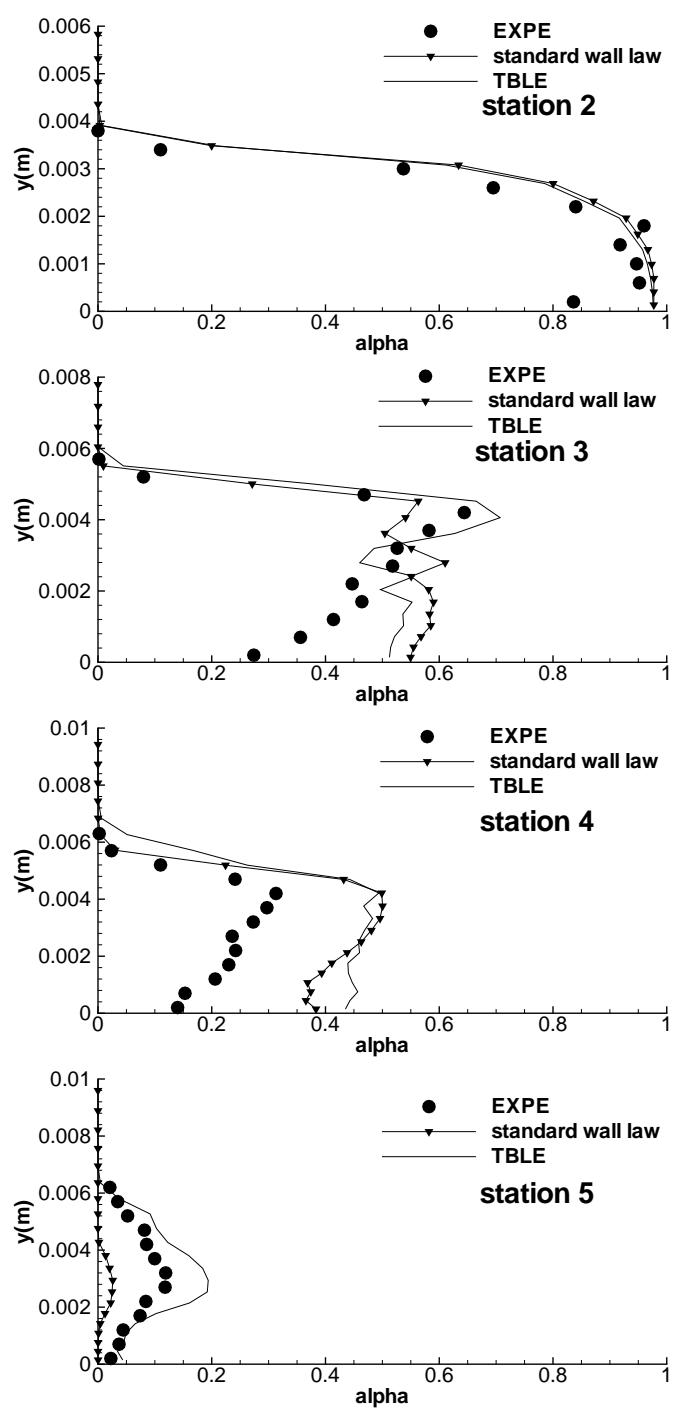

Figure 19: Time-averaged void ratio profiles from station 2 to 5 , Smith $k-l$ model, TBLE versus standard wall law, $4^{\circ}$ Venturi. 


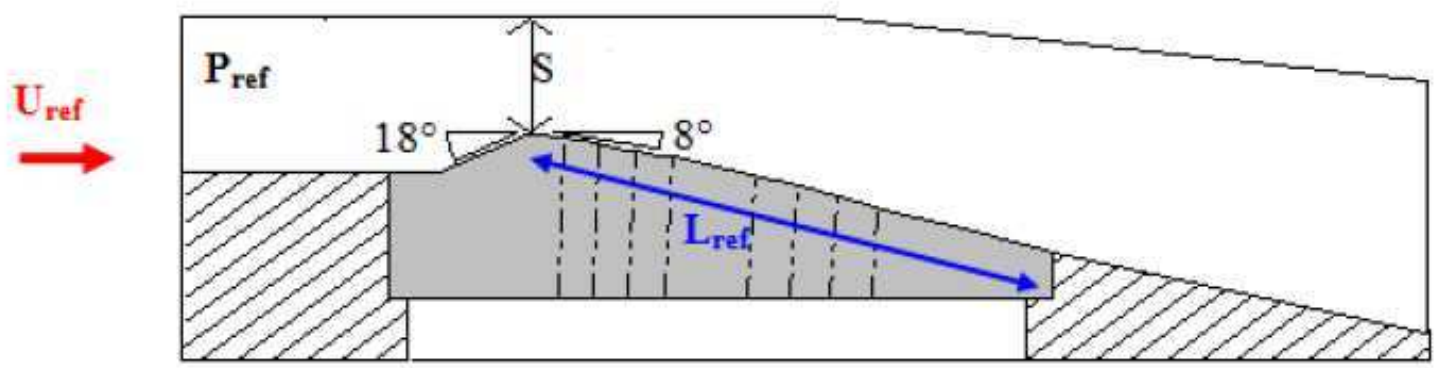

Figure 20: Schematic view of the $8^{\circ}$ Venturi profile. 


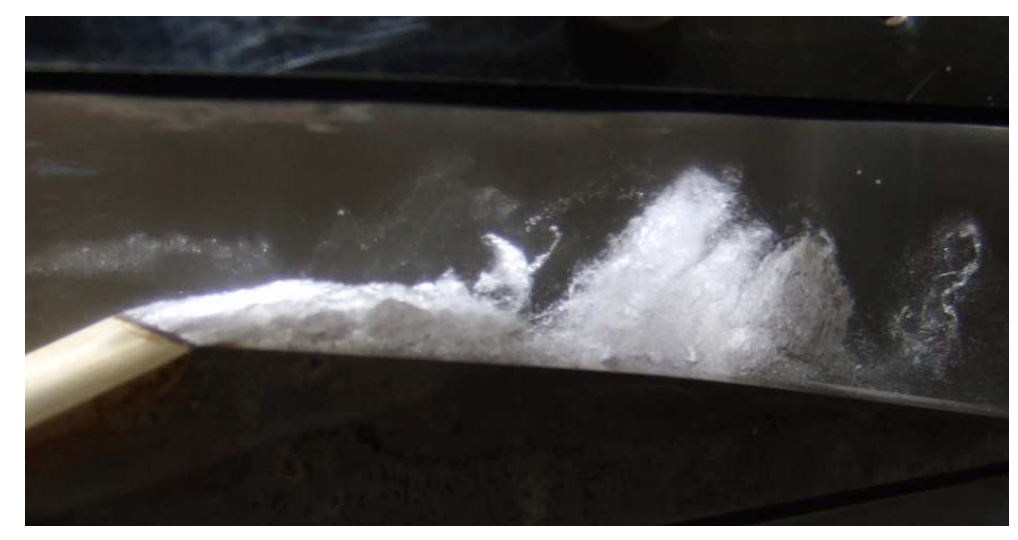

Figure 21: Photograph of the cavity, $8^{\circ}$ Venturi. 


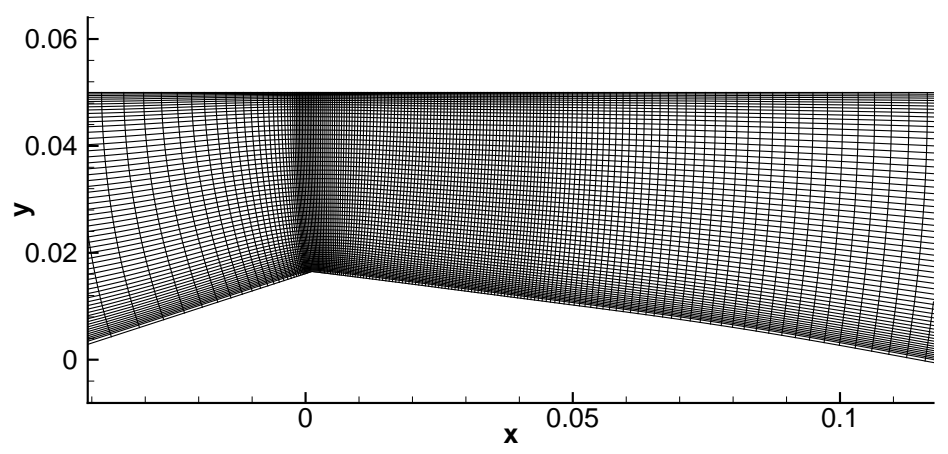

Figure 22: Enlargement of the mesh $174 \times 62$ near the throat, $8^{\circ}$ Venturi. 

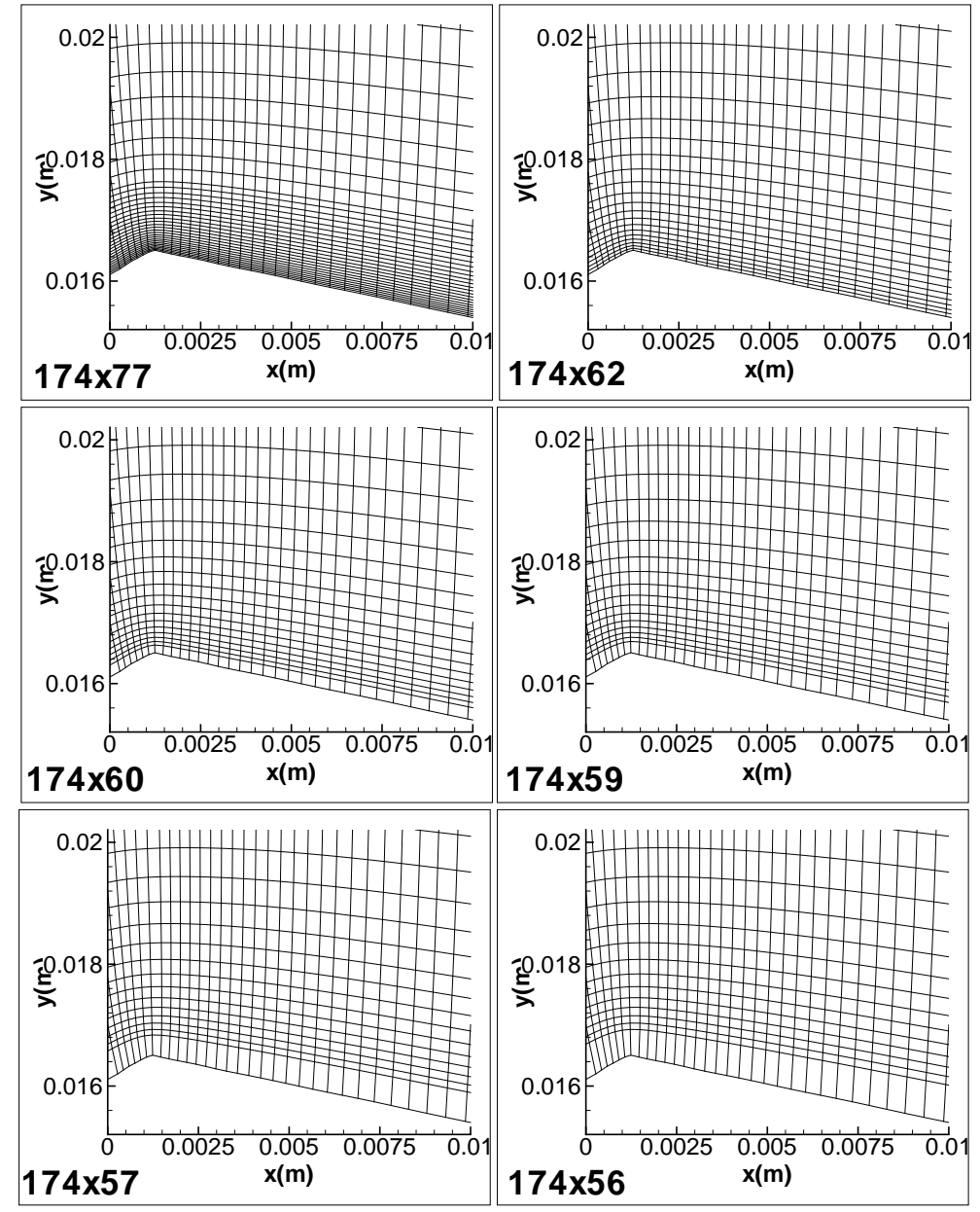

Figure 23: Enlargement of all meshes near the throat, $8^{\circ}$ Venturi. 


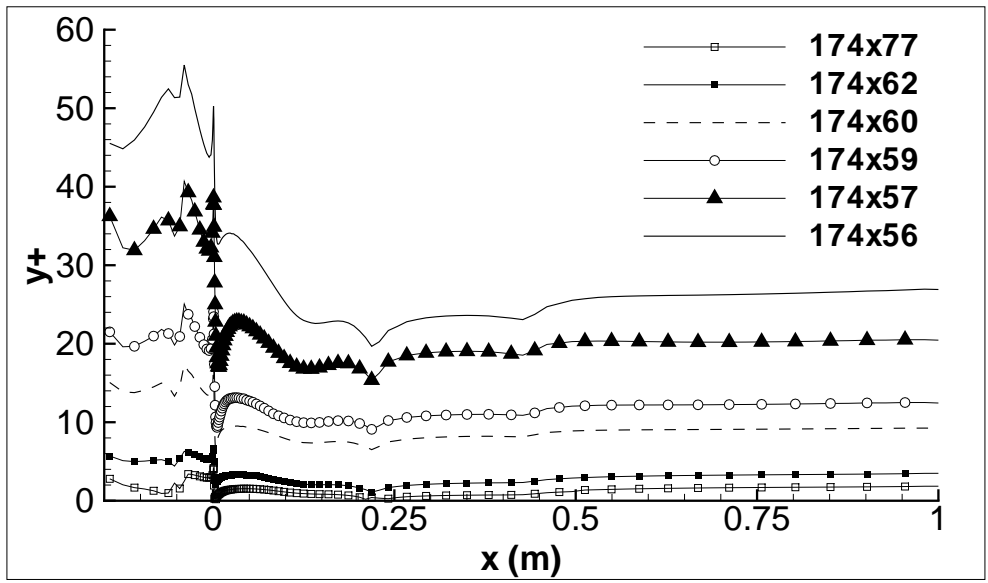

Figure 24: Evolution of the $y^{+}$values at the bottom wall, $8^{\circ}$ Venturi. 

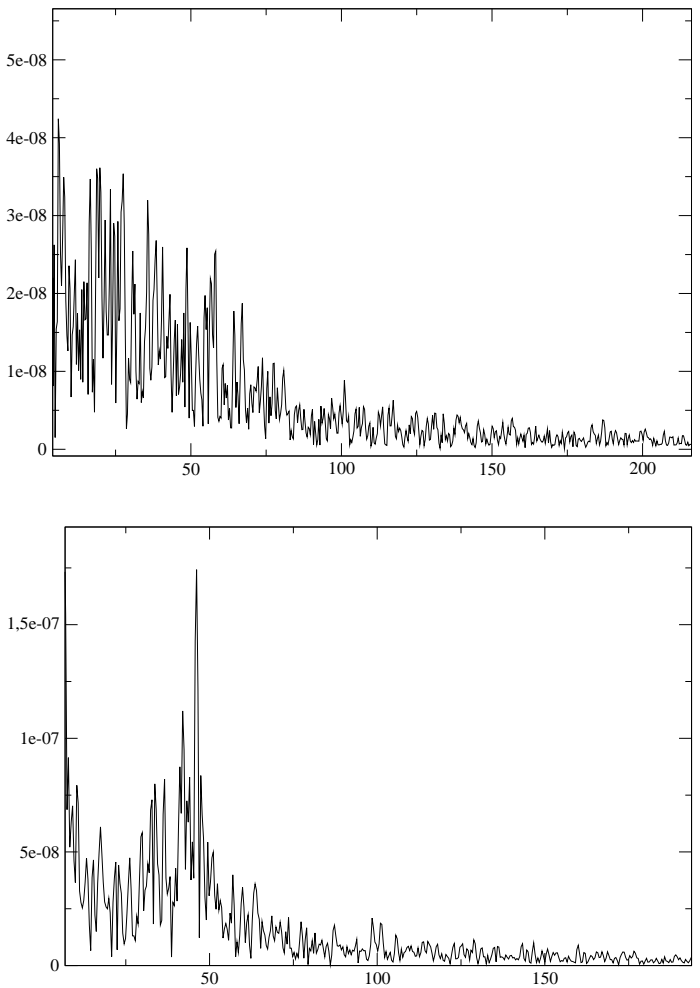

Figure 25: Vapour volume Fourier transformation, frequency (Hz), fine mesh 174x77 (top), coarse mesh 174x57 (down), $8^{\circ}$ Venturi. 

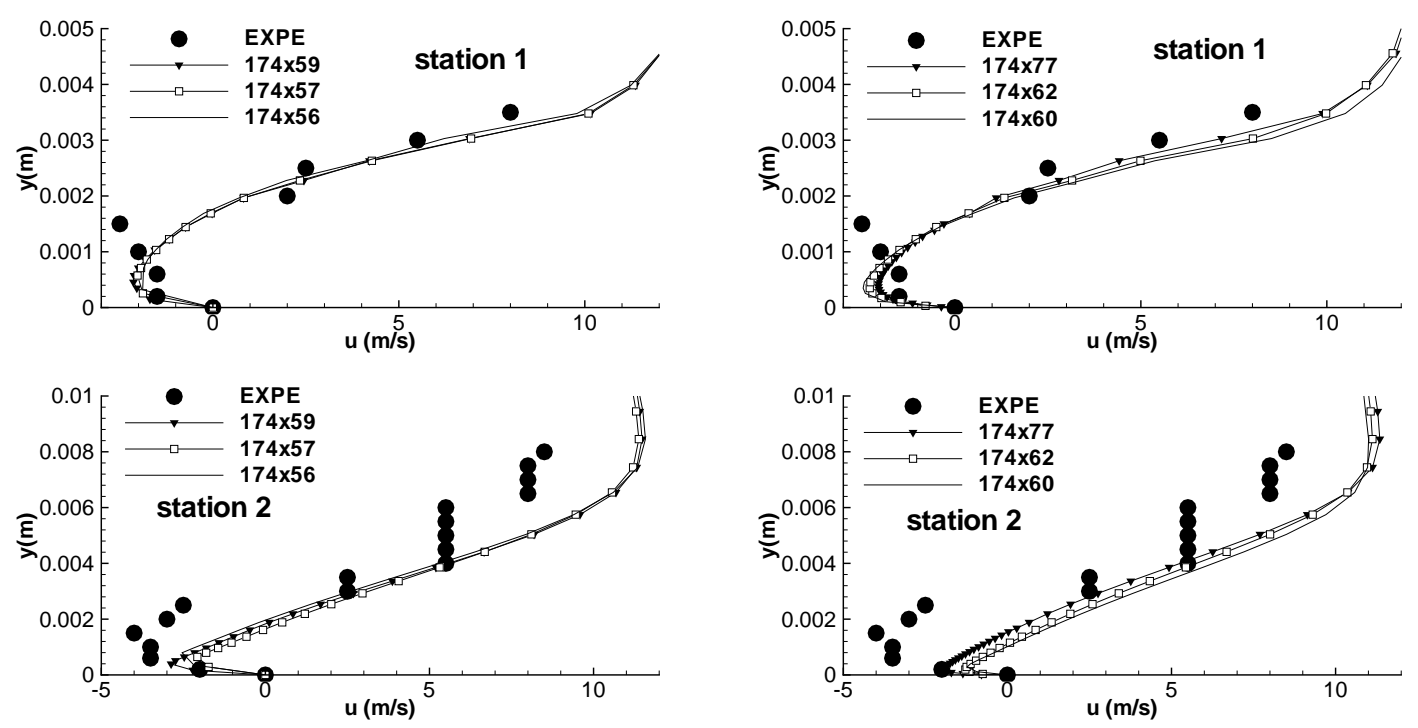

Figure 26: Time-averaged velocity profiles at station 1 and 2, coarsest meshes (left) versus finest meshes (right), $8^{\circ}$ Venturi. 

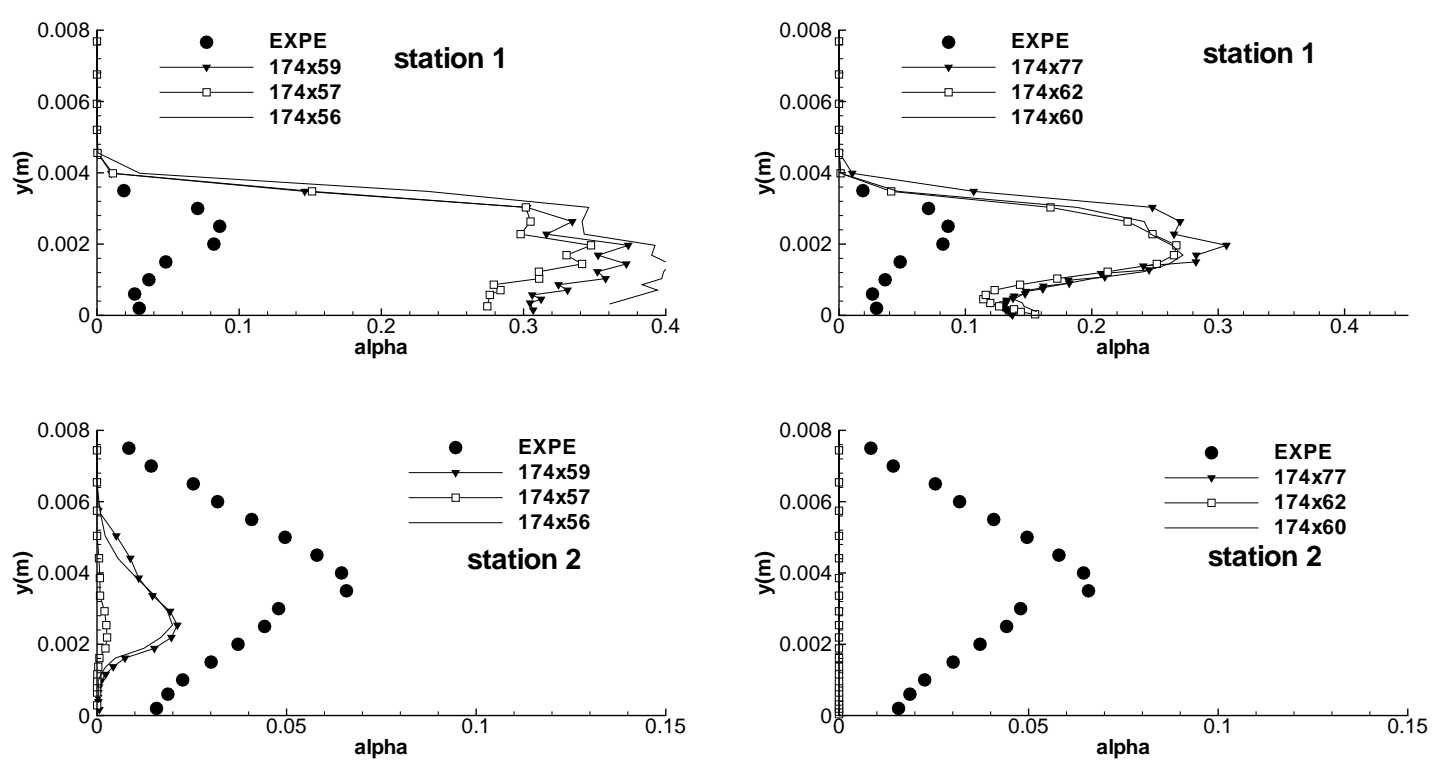

Figure 27: Time-averaged void ratio profiles at station 1 and 2, coarsest meshes (left) versus finest meshes (right), $8^{\circ}$ Venturi. 

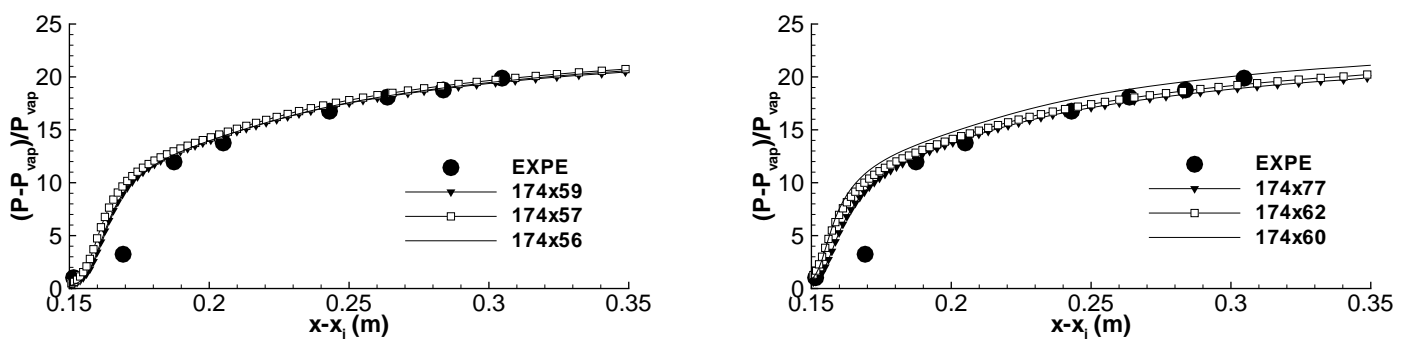

Figure 28: Time-averaged wall pressure evolution, coarsest meshes (left) versus finest meshes (right), $8^{\circ}$ Venturi. 

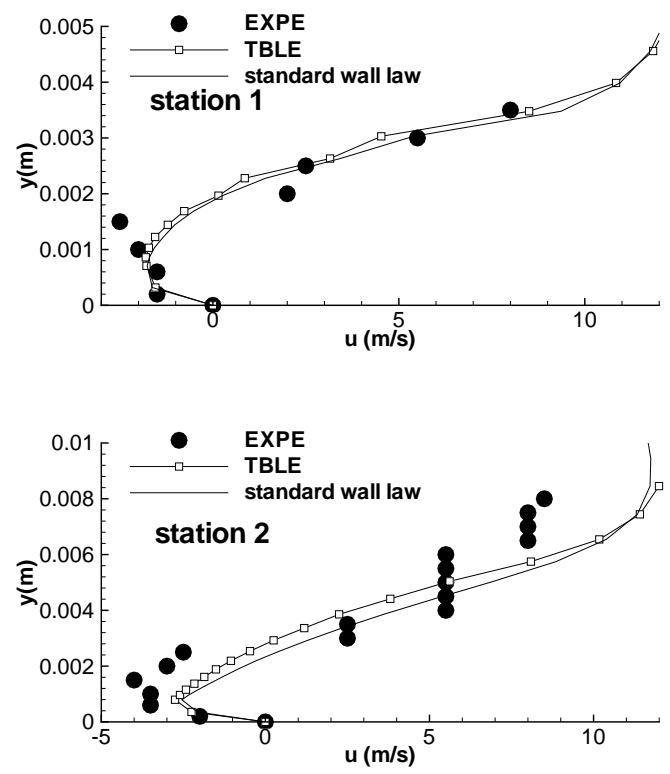

Figure 29: Time-averaged velocity profiles at station 1 and 2, TBLE versus standard wall law, $8^{\circ}$ Venturi. 

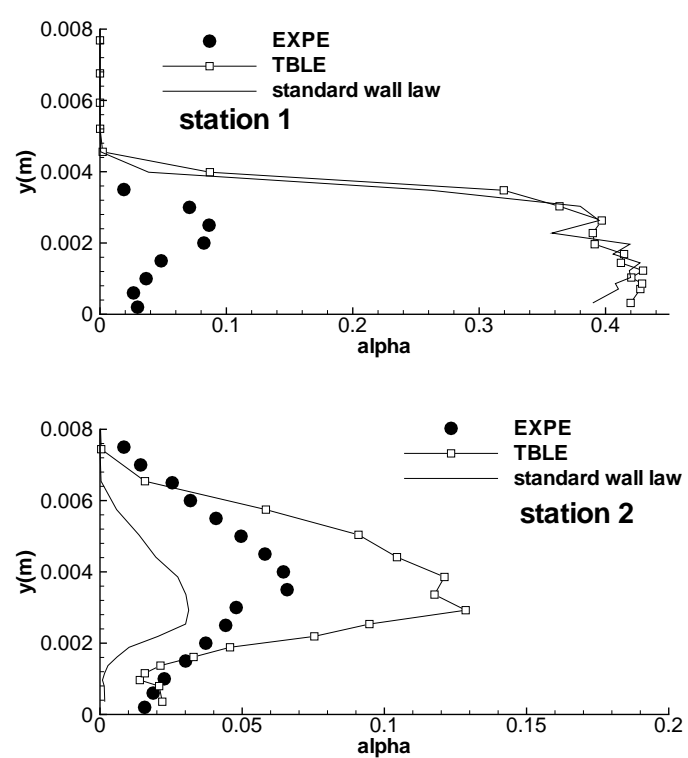

Figure 30: Time-averaged void ratio profiles at station 1 and 2, TBLE versus standard wall law, $8^{\circ}$ Venturi. 


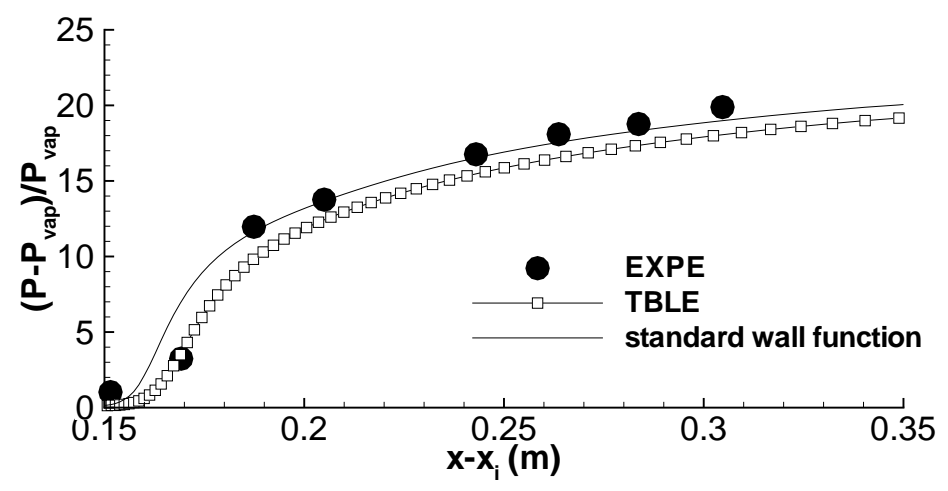

Figure 31: Time-averaged wall pressure evolution, TBLE versus standard wall law, $8^{\circ}$ Venturi. 